\title{
THE INFLUENCE OF THE FIRST TERM OF AN ARITHMETIC PROGRESSION
}

\author{
DANIEL FIORILLI
}

\begin{abstract}
The goal of this article is to study the discrepancy of the distribution of arithmetic sequences in arithmetic progressions. We will fix a sequence $\mathcal{A}=\{\mathbf{a}(n)\}_{n \geq 1}$ of non-negative real numbers in a certain class of arithmetic sequences. For a fixed integer $a \neq 0$, we will be interested in the behaviour of $\mathcal{A}$ over the arithmetic progressions $a \bmod q$, on average over $q$. Our main result is that for certain sequences of arithmetic interest, the value of $a$ has a significant influence on this distribution, even after removing the first term of the progressions.
\end{abstract}

\section{Contents}

1. Introduction

1.1. Acknowledgements

2. Examples

2.1. Primes

2.2. Integers represented by a fixed binary quadratic form, with multiplicity

2.3. Sums of two squares, without multiplicity

2.4. Prime $k$-tuples

2.5. Integers free of small prime factors

3. Definitions and Hypotheses

3.1. Arithmetic sequences

3.2. Hypotheses

3.3. The formula for the average

4. Main result

5. Proof of the main result

5.1. An estimate for the main sum

5.2. Proofs of theorems 4.1 and $4.1^{*}$ 
Appendix A. Generalities on binary quadratic forms 445

References

\section{INTRODUCTION}

The study of arithmetic sequences is a central problem in number theory. Undoubtedly, it is the sequence of prime numbers which has attracted the most attention amongst number theorists, leading to many theorems and conjectures. Other important sequences include sums of two squares, twin primes, divisor sequences and so on. In general, number theorists are interested in sequences with arithmetical content, and one can formally define wide classes of such sequences. Some phenomena occurring in the theory of prime numbers happen to be true for much wider classes of arithmetic sequences, such as the Bombieri-Vinogradov theorem for example (see [14]). Another example is the Granville-Soundararajan uncertainty principle (see [8]).

We will fix an integer $a \neq 0$ and study the distribution of an arithmetic sequence $\mathcal{A}=$ $\{\mathbf{a}(n)\}_{n \geq 1}$ in the progressions $a \bmod q$, on average over $q$. Under certain hypotheses, we will show how certain sequences remember the first term, that is how the value of $a$ can influence the distribution of $\mathcal{A}$ in the progressions $a \bmod q$. Examples of such sequences include the sequences of primes, sums of two squares (or more generally values of positive definite binary quadratic forms), prime $k$-tuples (conditionally) and integers without small prime factors. We will see that in each of these examples, values of $a$ which have the property that $\mathbf{a}(a)>0$ have a negative influence. More mysteriously, there are other values of $a$ having a negative influence, and it is not clear to me why these come up.

The structure of the paper will be as follows. We first give concrete examples to highlight the phenomena we want to describe. Then we give a framework to study arithmetic sequences, as well as the definitions which will be needed. We also state the hypotheses on which our main theorems will depend. We then state the main results, leaving the proofs to the final chapters. 
1.1. Acknowledgements. I would like to thank my supervisor Andrew Granville for suggesting this generalization and for his help and advice in general. I would also like to thank my colleagues Farzad Aryan, Mohammad Bardestani, Dimitri Dias, Tristan Freiberg and Kevin Henriot for many fruitful conversations. Ce travail a été rendu possible grâce à des bourses doctorales du Conseil de Recherche en Sciences Naturelles et en Génie du Canada et de la Faculté des Études Supérieures et Postdoctorales de l'Université de Montréal.

\section{EXAmpleS}

Before we state the general result, let us look at concrete examples. Throughout, $\mathcal{A}=$ $\{\mathbf{a}(n)\}_{n \geq 1}$ will be a fixed sequence of non-negative real numbers and $a \neq 0$ will be a fixed integer, on which every error term can possibly depend. We will adopt the convention that for negative values of $a, \mathbf{a}(a):=0$ (and similarly for $\Lambda(a)$ ). Moreover, $M=M(x)$ will denote a function tending to infinity with $x$, and we will use $\sim$ as shorthand for $\sim_{M \rightarrow \infty}$ (similarly for $\left.o(\cdot)=o_{M \rightarrow \infty}(\cdot)\right)$. We define the following counting functions.

\section{Definition 2.1.}

$$
\mathcal{A}(x):=\sum_{1 \leq n \leq x} \mathbf{a}(n), \quad \mathcal{A}_{d}(x):=\sum_{\substack{1 \leq n \leq x: \\ d \mid n}} \mathbf{a}(n), \quad \mathcal{A}(x ; q, a):=\sum_{\substack{1 \leq n \leq x \\ n \equiv a \bmod q}} \mathbf{a}(n)
$$

2.1. Primes. The first example we give, which was studied more precisely in [7], is the sequence of prime numbers.

Theorem 2.2. Let $A>0$ be a fixed real number. We have for $M=M(x) \leq(\log x)^{A}$ that

$$
\frac{1}{\frac{\phi(a)}{a} \frac{x}{M}} \sum_{\substack{q \leq \frac{x}{M} \\(q, a)=1}}\left(\psi(x ; q, a)-\Lambda(a)-\frac{\psi(x)}{\phi(q)}\right) \quad \text { is } \quad \begin{cases}\sim-\frac{1}{2} \log M & \text { if } a= \pm 1 \\ \sim-\frac{1}{2} \log p & \text { if } a= \pm p^{e} \\ =O_{\epsilon}\left(M^{-\frac{205}{538}+\epsilon}\right) & \text { otherwise. }\end{cases}
$$

2.2. Integers represented by a fixed binary quadratic form, with multiplicity. The second example we consider is the sequence of integers which can be represented by a fixed binary quadratic form $Q(x, y)$ with integer coefficients, counted with multiplicity, that is

$$
\mathbf{a}(n):=\#\left\{(x, y) \in \underset{3}{\mathbb{Z}_{\geq 0}^{2}}: Q(x, y)=n\right\}
$$


We will define $r_{d}(n)$ to be the total number of distinct representations of $n$ by all of the inequivalent forms of discriminant $d$ (which is not to be confused with $\mathbf{a}(n)$ ). By distinct representations, we mean that we count the representations up to automorphisms of the forms. We also define the function

$$
\rho_{a}(q):=\frac{1}{q} \cdot \#\{1 \leq x, y \leq q: Q(x, y) \equiv a \bmod q\}
$$

Theorem 2.3. Suppose that $Q(x, y)=\alpha x^{2}+\beta x y+\gamma y^{2}$ is a fixed positive definite quadratic form (with integer coefficients) of discriminant $d:=\beta^{2}-4 \alpha \gamma<0$, with $d \equiv 1,5,9,12,13 \bmod$ 16 (for simplicity). Fix an integer a such that $(a, 2 d)=1$. We have for $M=M(x) \leq x^{\lambda}$, where $\lambda<\frac{1}{12}$ is a fixed real number, that

$$
\frac{1}{x / M} \sum_{q \leq \frac{x}{M}}\left(\mathcal{A}(x ; q, a)-\mathbf{a}(a)-\frac{\rho_{a}(q)}{q} \mathcal{A}(x)\right)=-C_{Q} \rho_{a}(4 d) r_{d}(|a|)+O_{\epsilon}\left(\frac{1}{M^{1 / 3-\epsilon}}\right)
$$

with

$$
C_{Q}:=\frac{A_{Q}}{2 L\left(1, \chi_{d}\right)} \quad\left(=\frac{w_{d} \sqrt{|d|}}{4 \pi h_{d}} A_{Q}\right)
$$

where $A_{Q}$ is the area of the region $\left\{(x, y) \in \mathbb{R}_{\geq 0}^{2}: Q(x, y) \leq 1\right\}, \chi_{d}:=\left(\frac{4 d}{.}\right)$, w ${ }_{d}$ is the number of units of $\mathbb{Q}(\sqrt{d})$ and $h_{d}$ is its class number.

Remark. The number $\rho_{a}(4 d)$ is either zero or equal to $2^{\omega(2 d)}, 2^{\omega(2 d)-2}$ or $3 \cdot 2^{\omega(2 d)-2}$, depending on $Q(x, y)(\omega(n)$ denotes the number of prime factors of $n)$. For this reason, if $\rho_{a}(4 d)>0$, then it is independent of $a$.

Therefore, there is no bias if $\rho_{a}(4 d)=0$ or if $|a|$ cannot be represented by a form of discriminant $d$. However, if this is not the case, then the bias is proportional to the number of such representations.

2.3. Sums of two squares, without multiplicity. The next example is the sequence of integers which can be written as the sum of two squares, without multiplicity. We define

$$
\mathbf{a}(n):= \begin{cases}1 & \text { if } n=\square+\square \\ 0 & \text { else } \\ & 4\end{cases}
$$


For a fixed odd integer $a$, we define the multiplicative function $\mathbf{g}_{a}(q)$ on prime powers as follows. For $p \neq 2$ such that $p^{f} \| a$ with $f \geq 0$,

$$
\mathbf{g}_{a}\left(p^{e}\right):=\frac{1}{p^{e}} \times \begin{cases}1 & \text { if } p \equiv 1 \bmod 4 \\ 1 & \text { if } p \equiv 3 \bmod 4, e \leq f, 2 \mid e \\ \frac{1}{p} & \text { if } p \equiv 3 \bmod 4, e \leq f, 2 \nmid e \\ 1+\frac{1}{p} & \text { if } p \equiv 3 \bmod 4, e>f, 2 \mid f \\ 0 & \text { if } p \equiv 3 \bmod 4, e>f, 2 \nmid f .\end{cases}
$$

Moreover, $\mathbf{g}_{a}(2):=\frac{1}{2}$ and for $e \geq 2, \mathbf{g}_{a}\left(2^{e}\right):=\frac{1+\left(-1 \frac{a-1}{2}\right.}{2^{e+2}}$.

Theorem 2.4. Fix an integer $a \equiv 1 \bmod 4$. We have for $1 \leq M(x) \leq(\log x)^{\lambda}$, where $\lambda<1 / 5$ is a fixed real number, that

$$
\begin{aligned}
& \frac{1}{x / 2 M} \sum_{\frac{x}{2 M}<q \leq \frac{x}{M}}\left(\mathcal{A}(x ; q, a)-\mathbf{a}(a)-\mathbf{g}_{a}(q) \mathcal{A}(x)\right) \\
& \sim-\left(\frac{\log M}{\log x}\right)^{\frac{1}{2}} \frac{(-4)^{-l_{a}-1}\left(2 l_{a}+2\right) !}{\left(4 l_{a}^{2}-1\right)\left(l_{a}+1\right) ! \pi} \prod_{\substack{p^{f} \| a: \\
p \equiv 3 \text { mod } 4, f o d d}} \frac{\log \left(p^{\frac{f+1}{2}}\right)}{\log M},
\end{aligned}
$$

where $l_{a}:=\#\left\{p^{f} \| a: p \equiv 3 \bmod 4,2 \nmid f\right\}$ is the number primes dividing a to an odd power which are congruent to 3 modulo 4.

Remark. The right hand side of (3) is o((log $\left.x)^{-1 / 2}\right)$ iff $|a|$ cannot be written as the sum of two squares. Also, if $|a|=\square+\square$, then it is equal to $-\frac{1}{2 \pi}\left(\frac{\log M}{\log x}\right)^{\frac{1}{2}}$. Moreover, one can show that if $a \equiv 3 \bmod 4$, then the left hand side of (3) is always $o\left((\log x)^{-1 / 2}\right)$.

2.4. Prime $k$-tuples. The next example concerns prime $k$-tuples. Let $\mathcal{H}=\left\{\mathcal{L}_{1}, \ldots, \mathcal{L}_{k}\right\}$ be a $k$-tuple of distinct linear forms $\mathcal{L}_{i}(n)=a_{i} n+b_{i}$, with $a_{i}, b_{i} \in \mathbb{Z}, a_{i} \geq 1$, and define

$$
\mathcal{P}(n ; \mathcal{H}):=\prod_{\mathcal{L} \in \mathcal{H}} \mathcal{L}(n)
$$

We will suppose that $\mathcal{H}$ is admissible, that is for every prime $p$,

$$
\nu_{\mathcal{H}}(p):=\#\{x \bmod p: \underset{5}{\mathcal{P}}(x ; \mathcal{H}) \equiv 0 \bmod p\}<p
$$


Define

$$
\mathbf{a}(n):=\prod_{\mathcal{L} \in \mathcal{H}} \Lambda(\mathcal{L}(n))=\Lambda\left(a_{1} n+b_{1}\right) \Lambda\left(a_{2} n+b_{2}\right) \cdots \Lambda\left(a_{k} n+b_{k}\right)
$$

The singular series associated to $\mathcal{H}$ is

$$
\mathfrak{S}(\mathcal{H}):=\prod_{p}\left(1-\frac{\nu_{\mathcal{H}}(p)}{p}\right)\left(1-\frac{1}{p}\right)^{-k} .
$$

Note that if $(\mathcal{P}(a ; \mathcal{H}), q)>1$, then $\mathcal{A}(x ; q, a)$ is bounded. Fix $\delta>0$. The Hardy-Littlewood conjecture stipulates that there exists a function $\mathbf{L}(x)$ tending to infinity with $x$ such that if $(\mathcal{P}(a ; \mathcal{H}), q)=1$

$$
\mathcal{A}(x)=\mathfrak{S}(\mathcal{H}) x+O\left(\frac{x}{\mathbf{L}(x)^{2+2 \delta}}\right) .
$$

Define

$$
\gamma(q):=\prod_{p \mid q}\left(1-\frac{\nu_{\mathcal{H}}(p)}{p}\right) .
$$

Theorem 2.5. Assume that (4) holds uniformly for all admissible $k$-tuples $\mathcal{H}$ such that $\left|a_{i}\right| \leq \mathbf{L}(x)^{1+\delta}$ and $\left|b_{i}\right|=O(1)$. Then we have for $M=M(x) \leq \mathbf{L}(x)$ that the average

$$
\begin{aligned}
& \frac{1}{\frac{\phi(\mathcal{P}(a ; \mathcal{H}))}{\mathcal{P}(a ; \mathcal{H})} \frac{x}{2 M}} \sum_{\substack{\frac{x}{2 M}<q \leq \frac{x}{M}: \\
(q, \mathcal{P}(a ; \mathcal{H}))=1}}\left(\mathcal{A}(x ; q, a)-\mathbf{a}(a)-\frac{\mathcal{A}(x)}{q \gamma(q)}\right) \text { is } \\
& \begin{cases}\sim-\frac{(\log M)^{k-\omega(P(a ; \mathcal{H}))}}{2(k-\omega(\mathcal{P}(a ; \mathcal{H}))) !} \prod_{p \mid \mathcal{P}(a ; \mathcal{H})} \frac{p-\nu_{\mathcal{H}}(p)}{p-1} \log p & \text { if } \omega(\mathcal{P}(a ; \mathcal{H})) \leq k, \\
=O\left(\frac{1}{M^{\delta} k}\right) & \text { otherwise, }\end{cases}
\end{aligned}
$$

where $\delta_{k}>0$ is a positive real number depending on $k$, and $\omega(n)$ denotes the number of prime factors of $n$.

In the case of twin primes, we have $\mathcal{H}=\{n, n+2\}$, so $\mathcal{P}(a, \mathcal{H})=a(a+2)$, and the function $\nu_{\mathcal{H}}$ is given by $\nu_{\mathcal{H}}(2)=1$ and $\nu_{\mathcal{H}}(p)=2$ for odd $p$. We get that the average is

$$
\begin{cases}\sim-\frac{(\log M)^{2}}{4} & \text { if } a=-1 \\ \sim-\frac{\log 3}{4} \log M & \text { if } a=1,-3 \\ \sim-\frac{\log 2}{2} \log M & \text { if } a=2,-4 \\ \sim-\frac{\log p \log q}{2} \frac{p-\nu_{\mathcal{H}}(p)}{p-1} \frac{q-\nu_{\mathcal{H}}(q)}{q-1} & \text { if } a(a+2)= \pm p^{e} q^{f} \\ O\left(\frac{1}{M^{\delta_{2}}}\right) & \text { if } \omega(a(a+2)) \geq 3 .\end{cases}
$$


2.5. Integers free of small prime factors. For $y=y(x)$ a function of $x$, define

$$
\begin{array}{rlrl}
\mathbf{a}_{y}(n) & :=\left\{\begin{array}{cc}
1 & \text { if } p \mid n \Rightarrow p \geq y \\
0 & \text { else, }
\end{array}\right. & \mathcal{A}(x, y):=\sum_{n \leq x} \mathbf{a}_{y}(n), \\
\gamma_{y}(q):=\prod_{\substack{p \mid q \\
p<y}}\left(1-\frac{1}{p}\right), & \mathcal{A}(x, y ; q, a):=\sum_{\substack{n \leq x \\
n \equiv a \bmod q}} \mathbf{a}_{y}(n) .
\end{array}
$$

Theorem 2.6. Fix $a \neq 0, \delta>0$ and $M=M(x) \leq(\log x)^{1-\delta}$. If

$$
\nu_{y}(a, M):=\frac{1}{\frac{x}{2 M} \frac{\phi(a)}{a}} \sum_{\substack{\frac{x}{2 M}<q \leq \frac{x}{M} \\(q, a)=1}}\left(\mathcal{A}(x, y ; q, a)-\mathbf{a}_{y}(a)-\frac{\mathcal{A}(x, y)}{q \gamma_{y}(q)}\right),
$$

then for $y \leq e^{(\log M)^{\frac{1}{2}-\delta}}$ with $y \rightarrow \infty$

$$
\nu_{y}(a, M)= \begin{cases}-\frac{1}{2}+o(1) & \text { if } a= \pm 1 \\ o(1) & \text { otherwise }\end{cases}
$$

and for $(\log x)^{\log \log \log x} \leq y \leq \sqrt{x}$

$$
\nu_{y}(a, M)=\frac{\mathcal{A}(x, y)}{x} \times \begin{cases}(1+o(1)) \log M & \text { if } a= \pm 1 \\ \log p+o(1) & \text { if } a= \pm p^{k} \\ o(1) & \text { otherwise. }\end{cases}
$$

(We have no result in the intermediate range.)

Remark. For $x$ large enough, $\mathbf{a}_{y}(a)=0$ unless $a= \pm 1$.

\section{Definitions And Hypotheses}

3.1. Arithmetic sequences. The goal of this section is to give a framework to study arithmetic sequences. This discussion is modeled on that in [8].

We wish to study the sequence $\mathcal{A}=\{\mathbf{a}(n)\}_{n \geq 1}$ in arithmetic progressions, therefore one of our goals will be to prove the existence of a multiplicative function $\mathbf{g}_{a}(q)$ such that

$$
\mathcal{A}(x ; q, a) \sim \mathbf{g}_{a}(q) \mathcal{A}(x)
$$

whenever $\mathbf{g}_{a}(q) \neq 0$. Let us give a heuristic way to do this with the help of an auxiliary multiplicative function $\mathbf{h}(d)$. First, denote by $\mathcal{S}$ a finite set of "bad primes", which are 
inherent to the sequence $\mathcal{A}$. We will assume that $\mathcal{A}$ is well distributed in the progressions $0 \bmod d$, that is there exists a multiplicative function $\mathbf{h}(d)$ such that for $(d, \mathcal{S})=1$,

$$
\mathcal{A}_{d}(x) \approx \frac{\mathbf{h}(d)}{d} \mathcal{A}(x) .
$$

The fact that $\mathbf{h}(d)$ is multiplicative can be rephrased as "the events that $\mathbf{a}(n)$ is divisible by coprime integers are independent". Let us also assume that

$$
\mathcal{A}(x ; q, a) \approx \frac{1}{\phi(q /(q, a))} \sum_{\substack{n \leq x: \\(q, n)=(q, a)}} \mathbf{a}(n)
$$

that is the sum is equally partitioned amongst the $\phi(q /(q, a))$ arithmetic progressions $b \bmod q$ with $(b, q)=(a, q)$. We then compute

$$
\begin{aligned}
\mathcal{A}(x ; q, a) \approx \frac{1}{\phi(q /(q, a))} \sum_{\substack{n \leq x \\
(q, n)=(q, a)}} \mathbf{a}(n) & =\frac{1}{\phi(q /(q, a))} \sum_{d \mid \frac{q}{(q, a)}} \mu(d) \mathcal{A}_{(q, a) d}(x) \\
& \approx \mathcal{A}(x) \frac{1}{\phi(q /(q, a))} \sum_{d \mid \frac{q}{(q, a)}} \mu(d) \frac{\mathbf{h}((q, a) d)}{(q, a) d}=\mathbf{g}_{a}(q) \mathcal{A}(x),
\end{aligned}
$$

where

$$
\mathbf{g}_{a}(q)=\mathbf{g}_{(a, q)}(q):=\frac{1}{\phi(q /(q, a))} \sum_{d \mid \frac{q}{(q, a)}} \mu(d) \frac{\mathbf{h}((q, a) d)}{(q, a) d}
$$

is a multiplicative function of $q$ which depends on $(q, a)$ (rather than depending on $a$ ). We have thus expressed the multiplicative function $\mathbf{g}_{a}(q)$ in terms of $\mathbf{h}(d)$. More explicitly, we have, when $p^{f} \| a($ with $(p a, \mathcal{S})=1)$, that

$$
\mathbf{g}_{a}\left(p^{e}\right)= \begin{cases}\frac{\mathbf{h}\left(p^{e}\right)}{p^{e}} & \text { if } e \leq f \\ \frac{1}{\phi\left(p^{e}\right)}\left(\mathbf{h}\left(p^{f}\right)-\frac{\mathbf{h}\left(p^{f+1}\right)}{p}\right) & \text { if } e>f .\end{cases}
$$

In particular, if $p \nmid a$,

$$
\mathbf{g}_{a}\left(p^{e}\right)=\frac{1}{\phi\left(p^{e}\right)}\left(1-\frac{\mathbf{h}(p)}{p}\right)
$$

Another way to write this is

$$
\mathcal{A}(x ; q, a) \approx \frac{\mathbf{f}_{a}(q)}{q \gamma(q)} \mathcal{A}(x)
$$

where

$$
\gamma(q):=\frac{\phi(q)}{q} \prod_{p \mid q}\left(1-\frac{\mathbf{h}(p)}{p}\right)^{-1}=\prod_{p} \frac{1-1 / p}{1-\mathbf{h}(p) / p}
$$


and $\mathbf{f}_{a}(q)$ is a multiplicative function defined by $\mathbf{f}_{a}(q):=\mathbf{g}_{a}(q) q \gamma(q)$. Note that for $(a, q)=1$, $\mathbf{f}_{a}(q)=1$.

3.2. Hypotheses. In the following, $\delta>0$ will denote a (small) fixed real number which will change from one statement to another. We will also fix an integer $a \neq 0$ with the property that $(a, \mathcal{S})=1$, where $\mathcal{S}$ is a finite set of bad primes. The function $\mathbf{L}:[0, \infty) \rightarrow[1, \infty)$ will be a given increasing smooth function such that $\mathbf{L}(x) \rightarrow \infty$ as $x \rightarrow \infty$ (think of $\mathbf{L}(x)$ as a power of $\log x)$. We now assume the existence of a multiplicative function $\mathbf{f}_{a}(q)=\mathbf{f}_{(a, q)}(q)$, depending on $(a, q)$, and of $\gamma(q) \neq 0$, which is independent of $a$ (as in Section 3.3), such that for any fixed $a \neq 0$ and $q \geq 1$,

$$
\mathcal{A}(x ; q, a) \sim \frac{\mathbf{f}_{a}(q)}{q \gamma(q)} \mathcal{A}(x)
$$

whenever $\mathbf{f}_{a}(q) \neq 0$. To simplify the notation, we will also assume the existence of a multiplicative function $\mathbf{h}(d)$ such that (5) holds (for $(q a, \mathcal{S})=1$ ).

Hypothesis 3.1. There exists a positive increasing function $\mathbf{R}(x)$ (think of $\mathbf{R}(x)$ as a small power of $x)$, with $\mathbf{L}(x)^{1+\delta} \leq \mathbf{R}(x) \leq \sqrt{x}$, such that

$$
\sum_{q \leq 2 \mathbf{R}(x)} \max _{y \leq x}\left|\mathcal{A}(y ; q, a)-\frac{\mathbf{f}_{a}(q)}{q \gamma(q)} \mathcal{A}(y)\right| \ll \frac{\mathcal{A}(x)}{\mathbf{L}(x)^{1+\delta}} .
$$

We will see later that if we use dyadic intervals, we can replace Hypothesis 3.1 by a weaker hypothesis.

Hypothesis 3.1 $^{*}$. We have

$$
\sum_{q \leq 2 \mathbf{L}(x)} \max _{y \leq x}\left|\mathcal{A}(y ; q, a)-\frac{\mathbf{f}_{a}(q)}{q \gamma(q)} \mathcal{A}(y)\right| \ll \frac{\mathcal{A}(x)}{\mathbf{L}(x)^{1+\delta}} .
$$

Hypothesis 3.2. For any $z=z(x)$ in the range $\frac{1}{\mathbf{L}(x)} \leq z(x) \leq 1+\frac{|a|}{x}$, we have

$$
\frac{\mathcal{A}(z x)}{\mathcal{A}(x)}=z+O\left(\frac{1}{\mathbf{L}(x)^{1+\delta}}\right) \text {. }
$$

Moreover, for $n \leq x$, we have the following bound:

$$
\mathbf{a}(n) \ll \frac{\mathcal{A}(x)}{\mathbf{L}(x)^{1+\delta}} .
$$


The next hypothesis is somewhat more specific to our analysis than the ones above, and it will allow us to use the analytic theory of zeta functions.

Hypothesis 3.3. There exists a real number $\mathbf{k} \geq 0$ such that the sum

$$
\sum_{p \notin \mathcal{S}} \frac{\mathbf{h}(p)-\mathbf{k}}{p}
$$

is convergent. More generally, for any real number $t$ and integer $n \geq 1$, we have

$$
\begin{gathered}
\sum_{\substack{p \leq x \\
p \notin \mathcal{S}}} \frac{\mathbf{h}(p)-\mathbf{k}}{p^{1+i t}} \leq(1 / 2-\delta) \log (|t|+2)+O(1), \\
\sum_{\substack{p \leq x \\
p \notin \mathcal{S}}} \frac{(\mathbf{h}(p)-\mathbf{k}) \log ^{n} p}{p^{1+i t}} \ll_{n, \epsilon}(|t|+2)^{\epsilon} .
\end{gathered}
$$

Finally, $\mathbf{h}(p)<p$ and for any $\epsilon>0$,

$$
\mathbf{h}(d) \ll_{\epsilon} d^{\epsilon}
$$

The final hypothesis will be useful when studying the full interval $1 \leq q \leq \frac{x}{M}$ rather than a dyadic one. It is not known for all the sequences we considered in Section 2; for this reason we used dyadic intervals in theorems $2.4,2.5$ and 2.6 ,

Hypothesis 3.4. With the same $\mathbf{R}(x)$ as in Hypothesis 3.1, we have

$$
\sum_{q \leq \frac{x}{\mathbf{R}(x)}}\left(\mathcal{A}^{*}(x ; q, a)-\frac{\mathbf{f}_{a}(q)}{q \gamma(q)} \mathcal{A}(x)\right) \ll \frac{\mathcal{A}(x)}{\mathbf{L}(x)^{1+\delta}},
$$

where $\mathcal{A}^{*}(x ; q, a)$ is defined as in (24).

3.3. The formula for the average. In this section we give a formula for the "average" $\mu_{\mathbf{k}}(a, M)$ which will appear in theorems 4.1 and $4.1^{*}$. The formula is rather complicated in its general form, however in concrete examples it can be seen that it reflects the nature of the sequence $\mathcal{A}$.

\section{Definition 3.5.}

$$
\omega_{\mathbf{h}}(a):=\#\left\{p^{f} \| \text { a with } f \geq 1: \mathbf{h}\left(p^{f}\right)=\mathbf{h}\left(p^{f+1}\right) / p\right\}
$$


Definition 3.6. Assume Hypothesis 3.3 and suppose that $\mathcal{S}=\emptyset$ (for simplicity). For an integer $a \neq 0$ and a real number $\mathbf{k} \geq 0$, we define

$$
\begin{gathered}
\mu_{\mathbf{k}}(a, M):=-\frac{1}{2} \frac{(\log M)^{1-\mathbf{k}-\omega_{\mathbf{h}}(a)}}{\Gamma\left(2-\mathbf{k}-\omega_{\mathbf{h}}(a)\right)} \prod_{\substack{p^{f} \| a: \\
\mathbf{h}\left(p^{f}\right)=\frac{\mathbf{h}\left(p^{f+1}\right)}{f \geq 0},}} \frac{1+\mathbf{h}(p)+\ldots+\mathbf{h}\left(p^{f}\right)}{(1-1 / p)^{\mathbf{k}-1}} \log p \\
\times \prod_{\substack{p^{f} \| a: \\
\mathbf{h}\left(p^{f}\right) \neq \frac{\mathbf{h}\left(p^{f+1}\right)}{f^{p}},}} \frac{\mathbf{h}\left(p^{f}\right)-\mathbf{h}\left(p^{f+1}\right) / p}{(1-1 / p)^{\mathbf{k}}} .
\end{gathered}
$$

Remark. The first product on the right hand side of (17) is a finite product, since a is fixed and $\mathbf{h}(p)<p$ for all $p$. The second product is convergent, since for $p \nmid$ a we have $\mathbf{h}\left(p^{f}\right)-\mathbf{h}\left(p^{f+1}\right) / p=1-\mathbf{h}(p) / p \approx 1-\mathbf{k} / p$. Of course both these statements rely on the assumption of Hypothesis 3.3 .

Remark. One sees that for integer values of $\mathbf{k}, \mu_{\mathbf{k}}(a, M)=0$ iff $\omega_{\mathbf{h}}(a) \geq 2-\mathbf{k}$, by the location of the poles of $\Gamma(s)$. Moreover, since these are the only poles, we have $\mu_{\mathbf{k}}(a, M) \neq 0$ whenever $\mathbf{k} \notin \mathbb{Z}$.

Remark. If $\mathcal{S} \neq \emptyset$, we can still give a formula for $\mu_{\mathbf{k}}(a, M)$, assuming we understand well $\mathbf{g}_{a}\left(p^{e}\right)$ with $p \in \mathcal{S}$. However, this would complicate the already lengthy definition of $\mu_{\mathbf{k}}(a, M)$, so we only give individual descriptions in the examples.

\section{MAin RESUlT}

The main result of the paper is a formula for the average value of the discrepancy $\mathcal{A}(x ; q, a)-\frac{\mathbf{f}_{a}(q)}{q \gamma(q)} \mathcal{A}(x)$, summed over $1 \leq q \leq Q$, with $Q$ large enough in terms of $x$.

Theorem 4.1. Assume that hypotheses 3.1, 3.2, 3.3 and 3.4 hold with $\mathcal{S}=\emptyset$ (for simplicity) and the function $\mathbf{L}(x)$. Fix an integer $a \neq 0$ and let $M=M(x)$ be a function of $x$ such that $1 \leq M(x) \leq \mathbf{L}(x)$. We have

$$
\sum_{q \leq \frac{x}{M}}\left(\mathcal{A}(x ; q, a)-\mathbf{a}(a)-\frac{\mathbf{f}_{a}(q)}{q \gamma(q)} \mathcal{A}(x)\right)=\frac{\mathcal{A}(x)}{M}\left(\mu_{\mathbf{k}}(a, M)(1+o(1))+O_{A}\left(\frac{1}{\log ^{A} M}\right)\right)
$$


where $\mathbf{a}(a)$ is the first term of $\mathcal{A}(x ; q, a)$ for positive a, and whenever a is negative, we set $\mathbf{a}(a)=0$.

We also give a dyadic version, which assumes a weaker form of Hypothesis [3.1, and does not assume Hypothesis 3.4 at all.

Theorem 4.1*. Assume that hypotheses $3.1{ }^{*}$, 3.2 and 3.3 hold with $\mathcal{S}=\emptyset$ (for simplicity) and the function $\mathbf{L}(x)$. Fix an integer $a \neq 0$ and let $M=M(x)$ be a function of $x$ such that $1 \leq M(x) \leq \mathbf{L}(x)$. We have

$$
\sum_{\frac{x}{2 M}<q \leq \frac{x}{M}}\left(\mathcal{A}(x ; q, a)-\mathbf{a}(a)-\frac{\mathbf{f}_{a}(q)}{q \gamma(q)} \mathcal{A}(x)\right)=\frac{\mathcal{A}(x)}{2 M}\left(\mu_{\mathbf{k}}(a, M)(1+o(1))+O_{A}\left(\frac{1}{\log ^{A} M}\right)\right) .
$$

Remark 4.2. As we have seen in the examples of Section 2, theorems 4.1 and $4.1^{*}$ easily generalize to arbitrary (given) sets $\mathcal{S} \neq \emptyset$, as long as we understand $\mathbf{g}_{a}\left(p^{e}\right)$ for each $p \in \mathcal{S}$.

Remark 4.3. If $\mu_{\mathbf{k}}(a, M) \neq 0$, then theorems 4.1 and $4.1^{*}$ give asymptotics for the sum on the left hand side.

Remark 4.4. Suppose that $\mathbf{k}=0$ (e.g. when $\mathcal{A}$ is the sequence of primes).

If $\omega_{\mathbf{h}}(a) \geq 2$, then $\mu_{0}(a, M)=0$.

If $\omega_{\mathbf{h}}(a)=1$, so there is a unique $p_{0}^{f_{0}} \| a, f_{0} \geq 1$, such that $\mathbf{h}\left(p_{0}^{f_{0}}\right)=\mathbf{h}\left(p_{0}^{f_{0}+1}\right) / p_{0}$, then

$$
\mu_{0}(a, M)=-\frac{1}{2}\left(1-\frac{1}{p_{0}}\right)\left(1+\mathbf{h}\left(p_{0}\right)+\ldots+\mathbf{h}\left(p_{0}^{f_{0}}\right)\right) \log p_{0} \prod_{\substack{p^{f} \| a \\ f \geq 0 \\ p \neq p_{0}}}\left(\mathbf{h}\left(p^{f}\right)-\mathbf{h}\left(p^{f+1}\right) / p\right) .
$$

If $\omega_{\mathbf{h}}(a)=0$, then

$$
\mu_{0}(a, M)=-\frac{\log M}{2} \prod_{\substack{p^{f} \| a \\ f \geq 0}}\left(\mathbf{h}\left(p^{f}\right)-\mathbf{h}\left(p^{f+1}\right) / p\right) .
$$

Remark 4.5. Suppose that $\mathrm{k}=1$ (e.g. when $\mathcal{A}$ is the sequence of integers which can be written as the sum of two squares, counted with multiplicity). Then

$$
\mu_{1}(a, M)=-\frac{1}{2} \prod_{\substack{p^{f} \| a \\ f \geq 0}} \frac{\mathbf{h}\left(p^{f}\right)-\mathbf{h}\left(p^{f+1}\right) / p}{1-1 / p} .
$$


Remark 4.6. Suppose that $\mathbf{k}$ is an integer $\geq 2$ (e.g. when $\mathcal{A}$ is the sequence of integers of the form $\left(m+c_{1}\right)\left(m+c_{2}\right) \cdots\left(m+c_{\mathbf{k}}\right)$, where the $c_{i}$ are distinct integers $)$. Then $\mu_{1}(a, M)=0$.

\section{Proof of the main Result}

The goal of this section is to prove theorems 4.1 and $4.1^{\mathrm{k}}$.

5.1. An estimate for the main sum. In this section, we will assume that $\mathcal{S}=\emptyset$ for simplicity. Again, the results easily generalize to $\mathcal{S} \neq \emptyset$.

Proposition 5.1. Assume Hypothesis 3.3. Let $M=M(x)$ and $\mathbf{R}=\mathbf{R}(x)$ be two positive functions of $x$ such that $M(x)^{1+\delta} \leq \mathbf{R}(x) \leq \sqrt{x}$ for a fixed $\delta>0$. We have

$$
\begin{aligned}
\sum_{1 \leq r \leq \mathbf{R}} \frac{\mathbf{f}_{a}(r)}{r \gamma(r)}\left(1-\frac{r}{\mathbf{R}}\right)-\sum_{1 \leq r \leq M} & \frac{\mathbf{f}_{a}(r)}{r \gamma(r)}\left(1-\frac{r}{M}\right)-\sum_{\frac{x}{\mathrm{R}}<q \leq \frac{x}{M}} \frac{\mathbf{f}_{a}(q)}{q \gamma(q)} \\
= & \frac{\mu_{k}(a, M)}{M}\left(1+O\left(\frac{\log \log M}{\log M}\right)\right)+O_{A}\left(\frac{1}{M \log ^{A} M}\right) .
\end{aligned}
$$

The proof of Proposition 5.1 will require several lemmas.

Lemma 5.2. With $\mathbf{f}_{a}(n)$ and $\gamma(n)$ defined as in Section 3.1, we have

$$
\frac{\mathbf{f}_{a}(n)}{n \gamma(n)} \ll \frac{1}{\phi(n)} .
$$

Proof. By definition,

$$
\begin{aligned}
\frac{\mathbf{f}_{a}(n)}{n \gamma(n)}=\mathbf{g}_{a}(n) & =\prod_{p^{e} \| n} \mathbf{g}_{a}\left(p^{e}\right) \ll_{a, \mathcal{S}} \prod_{\substack{p^{e} \| n \\
p \nmid a, p \notin \mathcal{S}}} \mathbf{g}_{a}\left(p^{e}\right) \\
& =\prod_{\substack{p^{e} \| n \\
p \nmid a, p \notin \mathcal{S}}} \frac{1}{\phi\left(p^{e}\right)}\left(1-\frac{\mathbf{h}(p)}{p}\right) \\
& \leq \prod_{\substack{p^{e} \| n \\
p \nmid a, p \notin \mathcal{S}}} \frac{1}{\phi\left(p^{e}\right)} \ll_{a, \mathcal{S}} \frac{1}{\phi(n)} .
\end{aligned}
$$


Lemma 5.3. Assume Hypothesis [3.3. Let $h:[0, \infty) \rightarrow[0, \infty)$ be a piecewise continuous function supported on $[0,1]$, taking a value halfway between the limit values at discontinuities, and suppose the integral

$$
\mathcal{M} h(s):=\int_{0}^{1} h(x) x^{s-1} d x
$$

converges absolutely for $\Re(s)>0$. Then,

$$
\sum_{n \leq M} \frac{\mathbf{f}_{a}(n)}{n \gamma(n)} h\left(\frac{n}{M}\right)=\frac{1}{2 \pi i} \int_{(1)} \mathfrak{S}_{2}(s) \zeta(s+1) \zeta(s+2)^{1-\mathbf{k}} Z_{5}(s) \mathcal{M} h(s) M^{s} d s,
$$

where

$$
\begin{gathered}
\mathfrak{S}_{2}(s):=\prod_{\substack{p^{f} \| a \\
f \geq 1}}\left[\left(1+\frac{\mathbf{h}(p)}{p^{s+1}}+\ldots+\frac{\mathbf{h}\left(p^{f}\right)}{p^{f(s+1)}}\right)\left(1-\frac{1}{p^{s+1}}\right)\right. \\
\left.\quad+\frac{\mathbf{h}\left(p^{f}\right)-\mathbf{h}\left(p^{f+1}\right) / p}{1-1 / p} \frac{1}{p^{(f+1)(s+1)}}\right]\left(1-\frac{1}{p^{s+2}}\right)^{1-\mathbf{k}}, \\
Z_{5}(s):=\prod_{p \nmid a}\left(1+\frac{1}{p^{s+1}}\left(\frac{1}{\gamma(p)}-1\right)\right)\left(1-\frac{1}{p^{s+2}}\right)^{1-\mathbf{k}} .
\end{gathered}
$$

Moreover, $\mathfrak{S}_{2}(s)$ is holomorphic in $\mathbb{C} \backslash\{-2\}$ and $Z_{5}(s)$ is holomorphic for $\Re s>-1$.

Proof. Define

$$
Z_{\mathcal{A}}(s):=\sum_{n=1}^{\infty} \frac{\mathbf{g}_{a}(n)}{n^{s}}=\prod_{p}\left(1+\frac{\mathbf{g}_{a}(p)}{p^{s}}+\frac{\mathbf{g}_{a}\left(p^{2}\right)}{p^{2 s}}+\ldots\right) .
$$

A standard computation using the definition of $\mathbf{g}_{a}(n)$ (see (15)) yields that

$$
Z_{\mathcal{A}}(s)=\mathfrak{S}_{2}(s) \zeta(s+1) \zeta(s+2)^{1-\mathbf{k}} Z_{5}(s)
$$

The function $\mathfrak{S}_{2}(s)$ is clearly holomorphic in $\mathbb{C} \backslash\{-2\}$, and the fact that $Z_{5}(s)$ is holomorphic for $\Re s>-1$ follows from Hypothesis 3.3. Now, Mellin inversion gives that

$$
h\left(\frac{n}{M}\right)=\frac{1}{2 \pi i} \int_{(1)} \frac{M^{s}}{n^{s}} \mathcal{M} h(s) d s .
$$

Multiplying by $\frac{\mathbf{f}_{a}(n)}{n \gamma(n)}$ and summing over $n$ yields the result. 


\subsubsection{Properties of the Dirichlet series.}

Lemma 5.4. Assume Hypothesis 3.3. We have

$$
Z_{5}(s)=Z_{5}(-1)+O(|s+1|)
$$

in the region $|s+1| \leq 3$, with $\Re s>-1$.

Proof. We will show that

$$
\log \frac{Z_{5}(s)}{Z_{5}(-1)} \ll|s+1|,
$$

from which the lemma clearly follows. Let $s$ be a complex number with $\Re s>-1$. We compute

$$
\begin{aligned}
\log \frac{Z_{5}(s)}{Z_{5}(-1)} & =\sum_{p \nmid a} \log \left(\frac{1+\frac{1}{p^{s+1}}\left(\frac{1}{\gamma(p)}-1\right)}{\frac{1}{\gamma(p)}} \cdot \frac{\left(1-\frac{1}{p^{s+2}}\right)^{1-\mathbf{k}}}{\left(1-\frac{1}{p}\right)^{1-\mathbf{k}}}\right) \\
& =\sum_{p \nmid a}\left[\log \left(1-(1-\gamma(p))\left(1-\frac{1}{p^{s+1}}\right)\right)\right. \\
& =\sum_{p \nmid a}\left[\frac{\mathbf{h}(p)-1}{p-\mathbf{h}(p)}\left(1-\frac{1}{p^{s+1}}\right)+\frac{1-\mathbf{k}}{p-1}\left(1-\frac{1}{p^{s+1}}\right)\right] \\
& \left.=\sum_{p \nmid a}\left(\frac{\mathbf{h}(p)-1}{p-\mathbf{h}(p)}+\frac{1-\mathbf{k}}{p-1}\right)\left(1-\frac{1}{p-1}\left(1-\frac{1}{p^{s+1}}\right)\right)\right] \\
& =\sum_{p}\left(\frac{\mathbf{h}(p)-1}{p-\mathbf{h}(p)}+\frac{1-\mathbf{k}}{p-1}\right)\left(1-\frac{1}{p^{s+1}}\right)+O\left(|s+1|^{2} \sum_{p} \frac{\log ^{2} p}{p^{2-\epsilon}}\right)
\end{aligned}
$$

Note that by Hypothesis 3.3 , the series

$$
\sum_{p}\left(\frac{\mathbf{h}(p)-1}{p-\mathbf{h}(p)}+\frac{1-\mathbf{k}}{p-1}\right)=\sum_{p} \frac{\mathbf{h}(p)-\mathbf{k}}{p}+O(1)
$$


converges. Moreover, summation by parts yields the following estimate:

$$
S(t):=\sum_{p \leq t}\left(\frac{\mathbf{h}(p)-1}{p-\mathbf{h}(p)}+\frac{1-\mathbf{k}}{p-1}\right)=S(\infty)+O\left(\frac{1}{\log ^{2}(t+2)}\right) .
$$

We then get that

$$
\begin{aligned}
& \sum_{p \leq T}\left(\frac{\mathbf{h}(p)-1}{p-\mathbf{h}(p)}+\frac{1-\mathbf{k}}{p-1}\right)\left(1-\frac{1}{p^{s+1}}\right)=\int_{1}^{T}\left(1-\frac{1}{t^{s+1}}\right) d S(t) \\
& =\left.\left(1-\frac{1}{t^{s+1}}\right) S(t)\right|_{1} ^{T}-(s+1) \int_{1}^{T} \frac{S(t)}{t^{s+2}} d t \\
& =\left(1-\frac{1}{T^{s+1}}\right)\left(S(\infty)+O\left(\frac{1}{\log ^{2} T}\right)\right)-(s+1) \int_{1}^{T} \frac{S(\infty)}{t^{s+2}} d t \\
& \quad+O\left(|s+1| \int_{1}^{T} \frac{d t}{t \log ^{2}(t+2)}\right) \\
& =S(\infty)\left(1-\frac{1}{T^{s+1}}\right)+O\left(\frac{1}{\log ^{2} T}\right)+\left.\frac{S(\infty)}{t^{s+1}}\right|_{1} ^{T}+O(|s+1|) \\
& =O\left(\frac{1}{\log ^{2} T}+|s+1|\right) .
\end{aligned}
$$

Taking $T \rightarrow \infty$ yields that (12) is $\ll|s+1|$.

Lemma 5.5. Let $f(s)$ be a holomorphic function over a domain $\mathcal{D}$. We have that $\frac{f^{(n)}}{f}(s)$ is a polynomial in the variables $\left(\frac{f^{\prime}(s)}{f(s)}\right)^{(0)},\left(\frac{f^{\prime}(s)}{f(s)}\right)^{(1)}, \ldots,\left(\frac{f^{\prime}(s)}{f(s)}\right)^{(n-1)}$, with integer coefficients.

Proof. The proof goes by induction, using the identity

$$
\frac{f^{(n)}}{f}=\left(\frac{f^{(n-1)}}{f}\right)^{\prime}+\frac{f^{(n-1)}}{f} \frac{f^{\prime}}{f} .
$$

Lemma 5.6. Assume Hypothesis 3.3. Let $Z_{5}(s)$ be defined as in (111) and let $n \geq 0$. Then there exists $\delta>0$ such that, uniformly in the region $-1<\sigma<-\frac{1}{2}$ and $t \in \mathbb{R}$, we have

$$
Z_{5}^{(n)}(\sigma+i t) \ll_{n}(|t|+2)^{1 / 2-\delta}
$$

Proof. First write $Z_{5}(s)=Z_{3}(s) Z_{4}(s)$, where

$$
Z_{3}(s):=\prod_{p \nmid a}\left(1+\frac{1}{p^{s+1}}\left(\frac{1}{\gamma(p)}-1\right)\right)\left(1-\frac{1-\mathbf{k}}{p^{s+2}}\right),
$$




$$
Z_{4}(s):=\prod_{p \nmid a}\left(1-\frac{1}{p^{s+2}}\right)^{1-\mathbf{k}}\left(1-\frac{1-\mathbf{k}}{p^{s+2}}\right)^{-1} .
$$

The function $Z_{4}(s)$ is uniformly bounded in the region $\Re s \geq-1$, since the Eulerian product converges absolutely. As for $Z_{3}(s)$, we have for $-1<\sigma<-\frac{1}{2}$ that

$$
\log Z_{3}(\sigma+i t)=\log \prod_{p \nmid a}\left(1+\frac{1}{p^{\sigma+1+i t}} \frac{\mathbf{k}-\mathbf{h}(p)}{p}\right)+O(1) .
$$

Hypothesis 3.3 gives

$$
S(x, t):=\sum_{p \leq x} \frac{\mathbf{k}-\mathbf{h}(p)}{p^{1+i t}} \leq(1 / 2-\delta) \log (|t|+2)+O(1) .
$$

Thus,

$$
\begin{aligned}
\log \prod_{p \nmid a}\left(1+\frac{1}{p^{\sigma+1+i t}} \frac{\mathbf{k}-\mathbf{h}(p)}{p}\right) & =\sum_{p \nmid a} \frac{1}{p^{\sigma+1}} \frac{\mathbf{k}-\mathbf{h}(p)}{p^{1+i t}}+O(1) \\
& =\int_{1}^{\infty} \frac{d S(x, t)}{x^{\sigma+1}}+O(1) \\
& =\left.\frac{S(x, t)}{x^{\sigma+1}}\right|_{1} ^{\infty}+(\sigma+1) \int_{1}^{\infty} \frac{S(x, t)}{x^{\sigma+2}} d x+O(1) \\
& \leq(1 / 2-\delta) \log (|t|+2) \int_{1}^{\infty} \frac{\sigma+1}{x^{\sigma+2}} d x+O(1) \\
& =(1 / 2-\delta) \log (|t|+2)+O(1)
\end{aligned}
$$

which proves (13) for $n=0$. The bound

$$
\sum_{p \leq x} \frac{(\mathbf{k}-\mathbf{h}(p)) \log ^{m} p}{p^{1+i t}} \ll_{\epsilon}(|t|+2)^{\epsilon}
$$

gives

$$
\left(\frac{Z_{5}^{\prime}(\sigma+i t)}{Z_{5}(\sigma+i t)}\right)^{(m)} \ll_{\epsilon}(|t|+2)^{\epsilon}
$$

for $m \geq 0$. We finish the proof of (13) for $n \geq 1$ by applying Lemma 5.5.

Lemma 5.7. We have for $|\sigma+i t-1|>\frac{1}{10}$ that

$$
\zeta(\sigma+i t) \ll_{\epsilon}(|t|+2)^{\mu(\sigma)+\epsilon},
$$


where

$$
\mu(\sigma)= \begin{cases}1 / 2-\sigma & \text { if } \sigma \leq 0 \\ 1 / 2-2 \sigma / 3 & \text { if } 0 \leq \sigma \leq 1 / 2 \\ 1 / 3-\sigma / 3 & \text { if } 1 / 2 \leq \sigma \leq 1 \\ 0 & \text { if } \sigma \geq 1 .\end{cases}
$$

Moreover, these bounds are uniform for $\sigma$ contained in any compact subset of $\mathbb{R}$.

Proof. See Section II.3.4 of [17], in particular (II.3.13) and Theorem 3.8. By studying the proof of the Phragment-Lindelöf principle (see Chapter 9 of [6] for instance), we see that the bounds we get are uniform in $\sigma$.

Lemma 5.8. Assume Hypothesis 3.3. Let

$$
Z(s):=\frac{\mathfrak{S}_{2}(s) \zeta(s+1) \zeta(s+2)^{1-\mathbf{k}} Z_{5}(s)}{s(s+1)},
$$

with $\mathfrak{S}_{2}(s)$ and $Z_{5}(s)$ defined as in Lemma 5.3. There exists $\delta>0$ such that uniformly for $|t| \geq 2$ and $-1<\sigma<-\frac{1}{2}$,

$$
Z^{(n)}(\sigma+i t) \ll_{n} \frac{1}{|t|^{1+\delta}}
$$

Proof. Define

$$
Z_{6}(s):=\frac{\mathfrak{S}_{2}(s) \zeta(s+2)^{1-\mathbf{k}} Z_{5}(s)}{s(s+1)}
$$

Write $s=\sigma+i t$, with $-1<\sigma<-\frac{1}{2}$ and $|t| \geq 2$. We have for $m \geq 0$ that

$$
\left(\frac{Z_{6}^{\prime}(s)}{Z_{6}(s)}\right)^{(m)}=\left(\frac{\mathfrak{S}_{2}^{\prime}(s)}{\mathfrak{S}_{2}(s)}\right)^{(m)}+(1-\mathbf{k})\left(\frac{\zeta^{\prime}(s+2)}{\zeta(s+2)}\right)^{(m)}+\left(\frac{Z_{5}^{\prime}(s)}{Z_{5}(s)}\right)^{(m)}-\left(\frac{2 s+1}{s(s+1)}\right)^{(m)} .
$$

We compute that

$$
\left(\frac{\mathfrak{S}_{2}^{\prime}(s)}{\mathfrak{S}_{2}(s)}\right)^{(m)} \ll_{m} 1, \quad\left(\frac{2 s+1}{s(s+1)}\right)^{(m)} \ll_{m} 1, \quad\left(\frac{Z_{5}^{\prime}(s)}{Z_{5}(s)}\right)^{(m)} \ll_{m, \epsilon}|t|^{\epsilon} .
$$

(The first bound is clear, the second follows from the fact that $|t| \geq 2$ and the third comes from (14).) Applying Cauchy's formula for the derivatives as in Corollaire II.3.10 of [17] and then using the bound (II.3.55) of [17] yields

$$
\left(\frac{\zeta^{\prime}(s+2)}{\zeta(s+2)}\right)^{(m)} \ll_{m} \log ^{m+1}(|t|) .
$$


Using Lemma 5.5,

$$
Z_{6}^{(m)}(s) \ll_{\epsilon, m}\left|Z_{6}(s)\right||t|^{\epsilon}
$$

for $m \geq 0$. We now use Lemma 5.6 to bound $\left|Z_{5}(s)\right|$, which gives

$$
Z_{6}^{(m)}(s) \ll_{m}\left|\zeta(s+2)^{1-\mathbf{k}}\right||t|^{-3 / 2-2 \delta}
$$

for some $\delta>0$. Now if $\mathbf{k} \leq 1$, we use Lemma 5.7 to bound $\zeta(s+2)^{1-\mathbf{k}}$. Otherwise, we use the bound $(\zeta(s+2))^{-1} \ll \log (|t|)$ (see (II.3.56) of [17]). In both cases we get

$$
Z_{6}^{(m)}(s) \ll_{m}|t|^{-3 / 2-\delta}
$$

We now use Cauchy's formula for the derivatives, which states that

$$
\zeta^{(k)}(s+1)=\frac{k !}{2 \pi i} \oint_{|z|=r} \zeta(s+1+z) \frac{d z}{z^{k+1}} .
$$

Selecting $r=\epsilon / 2$ and applying Lemma 5.7, we get the bound]

$$
\zeta^{(k)}(s+1) \ll_{k, \epsilon}|t|^{1 / 2+\epsilon} .
$$

We conclude the existence of $\delta>0$ such that

$$
Z^{(n)}(s)=\sum_{i=0}^{n}\left(\begin{array}{l}
n \\
i
\end{array}\right) \zeta^{(i)}(s+1) Z_{6}^{(n-i)}(s) \ll_{n} \frac{1}{|t|^{1+\delta}}
$$

5.1.2. The value of $\mu_{\mathbf{k}}(a, M)$.

Proposition 5.9. Assume Hypothesis 3.3. If $\mathbf{k} \in \mathbb{Z}$, then

$$
\begin{aligned}
\frac{1}{2 \pi i} \int_{(-1 / 2)} \frac{\mathfrak{S}_{2}(s) \zeta(s+1) \zeta(s+2)^{1-\mathbf{k}} Z_{5}(s)}{s(s+1)} M^{s} d s=-\frac{\mu_{\mathbf{k}}(a, M)}{M}(1 & \left.+O\left(\frac{\log \log M}{\log M}\right)\right) \\
& +O_{A}\left(\frac{1}{M \log ^{A} M}\right)
\end{aligned}
$$

where $\mu_{\mathbf{k}}(a, M)$ is defined in Definition 3.6.

\footnotetext{
${ }^{1}$ This bound is still valid outside the zero-free region of $\zeta(s+1)$; this is why we considered the ordinary derivatives of $\zeta(s+1)$ instead of its logarithmic derivatives as with the other terms.
} 
Proof. We first need to understand the behaviour of

$$
\begin{aligned}
Z(s) & :=\frac{\mathfrak{S}_{2}(s) \zeta(s+1) \zeta(s+2)^{1-\mathbf{k}} Z_{5}(s)}{s(s+1)} \\
& =(s+1)^{\mathbf{k}+\omega_{\mathbf{h}}(a)-2} \frac{\mathfrak{S}_{2}(s)}{(s+1)^{\omega_{\mathbf{h}}(a)}} \zeta(s+1)((s+1) \zeta(s+2))^{1-\mathbf{k}} \frac{Z_{5}(s)}{s}
\end{aligned}
$$

in the region $\mathcal{D}:-1 \leq \Re s \leq-1 / 2$. This function is holomorphic for $\Re s>-1$ by Lemma 5.3, and as we will see, the only point in $\mathcal{D}$ where $Z(s)$ is not necessarily locally bounded is $s=-1$. The functions

$$
\zeta(s+1), \quad((s+1) \zeta(s+2))^{1-\mathbf{k}} \quad \text { and } \quad \frac{1}{s}
$$

are holomorphic on $\mathcal{D}$ and do not vanish at $s=-1$. The function $Z_{5}(s)$ is holomorphic for $\Re s>-1$, and all its derivatives are locally bounded around any point of $\mathcal{D}$ by Lemma 5.6, We compute

$$
Z_{5}(-1)=\prod_{p \nmid a} \frac{1-\mathbf{h}(p) / p}{(1-1 / p)^{\mathbf{k}}} \neq 0,
$$

since $\mathbf{h}(p)<p$. As for the function $\mathfrak{S}_{2}(s)$, it is holomorphic on $\mathcal{D}$. However, this function can vanish at $s=-1$ if for a certain $p \mid a$ we have $\mathbf{h}\left(p^{f}\right)=\mathbf{h}\left(p^{f+1}\right) / p$. In this case, we have for $s$ close to -1 that

$$
\begin{gathered}
\mathfrak{S}_{2}(s) \prod_{p \mid a}\left(1-\frac{1}{p^{s+2}}\right)^{\mathbf{k}-1}=\prod_{\substack{p^{f} \| a: \\
\mathbf{h}\left(p^{f} \neq \mathbf{h}\left(p^{f+1}\right) / p, f \geq 1\right.}}\left[\frac{\mathbf{h}\left(p^{f}\right)-\mathbf{h}\left(p^{f+1}\right) / p}{1-1 / p}+O(|s+1|)\right] \\
\times \prod_{\substack{p^{f} \| a: \\
\mathbf{h}\left(p^{f}\right)=\mathbf{h}\left(p^{f+1}\right) / p, f \geq 1}}\left[(s+1)\left(1+\mathbf{h}(p)+\ldots+\mathbf{h}\left(p^{f}\right)\right) \log p+O\left(|s+1|^{2}\right)\right],
\end{gathered}
$$

and since $\mathbf{h}\left(p^{e}\right) \geq 0$, this shows that every local factor has at most a simple zero at $s=-1$. We conclude that

$$
\frac{\mathfrak{S}_{2}(s)}{(s+1)^{\omega_{\mathbf{h}}(a)}}
$$

is holomorphic on $\mathcal{D}$ and does not vanish at $s=-1$. We now split in three distinct cases, depending on the analytic nature of $(s+1)^{\mathbf{k}+\omega_{\mathbf{h}}(a)-2}$ near $s=-1$. 
First case: $\mathbf{k}+\omega_{\mathbf{h}}(a) \geq 2$. In this case, $Z(s)$ and all of its derivatives are bounded near $s=-1$. To show this, note that it is true for the functions

$$
(s+1)^{\mathbf{k}+\omega_{\mathbf{h}}(a)-2}, \quad \frac{\mathfrak{S}_{2}(s)}{(s+1)^{\omega_{\mathbf{h}}(a)}}, \quad \zeta(s+1), \quad((s+1) \zeta(s+2))^{1-\mathbf{k}}, \quad \frac{1}{s} \quad \text { and } \quad Z_{5}(s),
$$

so it is also true for $Z(s)$ by Leibniz's rule. We now shift the contour of integration to the left until the line $\Re s=-1+\frac{1}{\log M}$ to get

$$
\begin{aligned}
\frac{1}{2 \pi i} \int_{(-1 / 2)} Z(s) d s & =\frac{i}{2 \pi i} \int_{\mathbb{R}} Z\left(-1+\frac{1}{\log M}+i t\right) M^{-1+\frac{1}{\log M}+i t} d t \\
& =\frac{e}{M} \frac{1}{2 \pi} \int_{\mathbb{R}} Z\left(-1+\frac{1}{\log M}+i t\right) e^{i t \log M} d t
\end{aligned}
$$

which gives, after $A$ integrations by parts,

$$
\begin{aligned}
\frac{1}{2 \pi i} \int_{(-1 / 2)} Z(s) d s & \ll_{A} \frac{1}{M \log ^{A} M} \int_{\mathbb{R}}\left|Z^{(A)}\left(-1+\frac{1}{\log M}+i t\right)\right|\left|e^{i t \log M}\right| d t \\
& \ll_{A} \frac{1}{M \log ^{A} M}\left(O(1)+\int_{|t| \geq 2} \frac{1}{|t|^{1+\delta}} d t\right) \\
& \ll_{A} \frac{1}{M \log ^{A} M}
\end{aligned}
$$

by Lemma 5.8. Note that the uniformity in $\sigma$ was crucial. This shows that we can take $\mu_{\mathbf{k}}(a, M)=0$.

Second case: $\mathbf{k}+\omega_{\mathbf{h}}(a)=1$. Let

$$
c:=\lim _{s \rightarrow 1^{+}}(s+1) Z(s) \neq 0
$$

and define

$$
Z_{7}(s):=Z(s)-\frac{c}{s+1}
$$

We can show using Lemma 5.4 that for $s$ close to -1 with $\Re s>-1$, the following bound holds:

$$
Z_{7}(s) \ll 1
$$

Lemma 5.4 implies that for $s$ close to -1 with $\Re s>-1$, the function

$$
Z_{7}^{\prime}(s)=\frac{((s+1) Z(s))^{\prime}}{s+1}-\frac{(s+1) Z(s)}{(s+1)^{2}}+\frac{c}{(s+1)^{2}}
$$

satisfies

$$
Z_{7}^{\prime}(s) \ll \frac{1}{|s+1|}
$$


Using Lemma 5.8, we get that for $|t| \geq 2$,

$$
Z_{7}^{\prime}(s) \ll \frac{1}{|t|^{1+\delta}}
$$

Thus,

$$
\begin{aligned}
\frac{1}{2 \pi i} \int_{\left(-1+\frac{1}{\log M}\right)} Z_{7}(s) M^{s} d s & =\frac{-1}{2 \pi i \log M} \int_{\left(-1+\frac{1}{\log M}\right)} Z_{7}^{\prime}(s) M^{s} d s \\
& \ll \frac{1}{M \log M}\left|\int_{-\infty}^{\infty} Z_{7}^{\prime}\left(-1+\frac{1}{\log M}+i t\right) M^{i t} d t\right| \\
& \ll \frac{1}{M \log M}\left(\left|\int_{-2}^{2} Z_{7}^{\prime}\left(-1+\frac{1}{\log M}+i t\right) M^{i t} d t\right|+O(1)\right) \\
& \ll \frac{1}{M \log M}\left(\int_{-2}^{2} \frac{1}{\frac{1}{\log M}+|t|} d t+O(1)\right) \\
& \ll \frac{1}{M \log M}\left(\int_{0}^{\frac{1}{\log M}} \log M+\int_{\frac{1}{\log M}}^{2} \frac{1}{t} d t+O(1)\right) \\
& \ll \frac{\log \log M}{M \log M} .
\end{aligned}
$$

Combining this bound with an easy residue computation yields

$$
\begin{aligned}
\frac{1}{2 \pi i} \int_{(-1 / 2)} Z(s) M^{s} d s & =\frac{1}{2 \pi i} \int_{(-1 / 2)} Z_{7}(s) M^{s} d s+\frac{1}{2 \pi i} \int_{(-1 / 2)} \frac{c}{s+1} M^{s} d s \\
& =\frac{c}{M}\left(1+O\left(\frac{\log \log M}{\log M}\right)\right) .
\end{aligned}
$$

Now remarks 4.4 and 4.5 show that $c=-\mu_{\mathbf{k}}(a, M)$, which concludes this case.

Third case: $\mathbf{k}=\omega_{\mathbf{h}}(a)=0$. Defining

$$
c:=\lim _{s \rightarrow-1^{+}}(s+1)^{2} Z(s) \neq 0,
$$

we get that the function $Z_{8}(s):=Z(s)-\frac{c}{(s+1)^{2}}$ satisfies the bound

$$
Z_{8}(s) \ll \frac{1}{|s+1|}
$$

by Lemma 5.4. An easy residue computation yields

$$
\frac{1}{2 \pi i} \int_{(-1 / 2)} Z(s) M^{s} d s=c \frac{\log M}{M}+\frac{1}{2 \pi i} \int_{\left(-1+\frac{1}{\log M}\right)} Z_{8}(s) M^{s} d s .
$$


Proceeding in an analogous way to the previous case, we compute

$$
\begin{aligned}
\int_{\left(-1+\frac{1}{\log M}\right)} Z_{8}(s) M^{s} d s & \ll \frac{1}{M}\left|\int_{-\infty}^{\infty} Z_{8}\left(-1+\frac{1}{\log M}+i t\right) M^{i t} d t\right| \\
& \ll \frac{1}{M}\left(\left|\int_{-2}^{2} Z_{8}\left(-1+\frac{1}{\log M}+i t\right) M^{i t} d t\right|+O(1)\right) \\
& \ll \frac{\log \log M}{M},
\end{aligned}
$$

from which we conclude

$$
\begin{aligned}
\frac{1}{2 \pi i} \int_{(-1 / 2)} Z(s) M^{s} d s & =c \frac{\log M}{M}\left(1+\frac{\log \log M}{\log M}\right) \\
& =-\frac{\mu_{0}(a, M)}{M}\left(1+\frac{\log \log M}{\log M}\right)
\end{aligned}
$$

by Remark 4.4, since

$$
c=\frac{1}{2} \prod_{\substack{p^{f} \| a \\ f \geq 0}}\left(\mathbf{h}\left(p^{f}\right)-\mathbf{h}\left(p^{f+1}\right) / p\right) .
$$

Lemma 5.10. Let $z>1$ be a real number. Then,

$$
\frac{1}{2 \pi i} \int_{\Re s=-1 / 2} \frac{M^{s}}{(s+1)^{z}} d s=\frac{1}{M} \frac{(\log M)^{z-1}}{\Gamma(z)} .
$$

Proof. Let $R \geq 2$ be a large real number and consider $\mathcal{H}_{R}$ a Hankel contour centered at $s=-1$ and truncated at $-R \pm \epsilon i$. Define $C_{R}$ to be the union of two circle segments starting at the endpoints of $\mathcal{H}_{R}$ and ending at the points $\pm i R$. By Cauchy's formula,

$$
\begin{aligned}
\frac{1}{2 \pi i} \int_{\Re_{s=-1 / 2}} \frac{M^{s}}{(s+1)^{z}} d s & =\frac{1}{2 \pi i} \int_{\Re_{s=0}} \frac{M^{s}}{(s+1)^{z}} d s \\
& =\frac{1}{2 \pi i} \int_{\mathcal{H}_{R}} \frac{M^{s}}{(s+1)^{z}} d s+\frac{1}{2 \pi i} \int_{C_{R}} \frac{M^{s}}{(s+1)^{z}} d s \\
& =\frac{1}{2 \pi i} \int_{\mathcal{H}_{R}} \frac{M^{s}}{(s+1)^{z}} d s+O\left(\frac{1}{R^{z-1}}\right),
\end{aligned}
$$

so by taking $R \rightarrow \infty$, 


$$
\begin{aligned}
\frac{1}{2 \pi i} \int_{\Re s=-1 / 2} \frac{M^{s}}{(s+1)^{z}} d s & =\frac{1}{2 \pi i} \int_{\mathcal{H}_{\infty}} \frac{M^{s}}{(s+1)^{z}} d s \\
& =\frac{1}{M} \frac{1}{2 \pi i} \int_{\mathcal{H}_{\infty}} \frac{e^{(s+1) \log M}}{(s+1)^{z}} d s \\
& =\frac{(\log M)^{z-1}}{M} \frac{1}{2 \pi i} \int_{\mathcal{H}_{\infty}^{\prime}} \frac{e^{w}}{w^{z}} d w \\
& =\frac{1}{M} \frac{(\log M)^{z-1}}{\Gamma(z)}
\end{aligned}
$$

by Hankel's formula (see Théorème II.0.17 of [17]). Here, $\mathcal{H}_{\infty}^{\prime}$ denotes an infinite Hankel contour centered at $w=0$.

Proposition 5.11. Assume Hypothesis [3.3. If $\mathbf{k} \notin \mathbb{Z}$, then

$$
\frac{1}{2 \pi i} \int_{(-1 / 2)} \frac{\mathfrak{S}_{2}(s) \zeta(s+1) \zeta(s+2)^{1-\mathbf{k}} Z_{5}(s)}{s(s+1)} M^{s} d s=-\frac{\mu_{\mathbf{k}}(a, M)}{M}\left(1+O\left(\frac{1}{\log M}\right)\right) .
$$

Proof. As in Proposition 5.9, we need to study the function

$$
Z(s)=(s+1)^{\mathbf{k}+\omega_{\mathbf{h}}(a)-2} \frac{\mathfrak{S}_{2}(s)}{(s+1)^{\omega_{\mathbf{h}}(a)}} \zeta(s+1)((s+1) \zeta(s+2))^{1-\mathbf{k}} \frac{Z_{5}(s)}{s}
$$

in the region $\mathcal{D}:-1 \leq \Re s \leq-1 / 2$. This function is holomorphic for $\Re s>-1$ by Lemma 5.6. and the only point in $\mathcal{D}$ where $Z(s)$ is not necessarily locally bounded is $s=-1$. However, the functions

$$
\frac{\mathfrak{S}_{2}(s)}{(s+1)^{\omega_{\mathbf{h}}(a)}}, \quad \zeta(s+1), \quad((s+1) \zeta(s+2))^{1-\mathbf{k}} \quad \text { and } \quad \frac{1}{s}
$$

are holomorphic on $\mathcal{D}$ and do not vanish at $s=-1$. The function $Z_{5}(s)$ is holomorphic for $\Re s>-1$, all its derivatives are locally bounded around any point of $\mathcal{D}$, and $Z_{5}(-1) \neq 0$. Define

$$
Z_{9}(s):=Z(s)-c(s+1)^{\mathbf{k}+\omega_{\mathbf{h}}(a)-2},
$$

where

$$
c:=\lim _{s \rightarrow-1^{+}}(s+1)^{2-\mathbf{k}-\omega_{\mathbf{h}}(a)} Z(s) \neq 0 .
$$


We have that

$$
\begin{aligned}
\frac{1}{2 \pi i} \int_{(-1 / 2)} Z(s) M^{s} d s= & \frac{(-1)^{\lceil\mathbf{k}\rceil+\omega_{\mathbf{h}}(a)}}{2 \pi i(\log M)^{\lceil\mathbf{k}\rceil+\omega_{\mathbf{h}}(a)}} \int_{(-1 / 2)} Z^{\left(\lceil\mathbf{k}\rceil+\omega_{\mathbf{h}}(a)\right)}(s) M^{s} d s \\
= & \frac{(-1)^{\lceil\mathbf{k}\rceil+\omega_{\mathbf{h}}(a)}}{2 \pi i(\log M)^{\lceil\mathbf{k}\rceil+\omega_{\mathbf{h}}(a)}}\left(\int_{(-1 / 2)} Z_{9}^{\left(\lceil\mathbf{k}\rceil+\omega_{\mathbf{h}}(a)\right)}(s) M^{s} d s\right. \\
& \left.\quad+c \frac{\Gamma\left(\mathbf{k}+\omega_{\mathbf{h}}(a)-1\right)}{\Gamma(\mathbf{k}-\lceil\mathbf{k}\rceil-1)} \int_{(-1 / 2)}(s+1)^{\mathbf{k}-\lceil\mathbf{k}\rceil-2} M^{s} d s\right) \\
=\frac{c}{M} \frac{(\log M)^{1-\mathbf{k}-\omega_{\mathbf{h}}(a)}}{\Gamma\left(2-\mathbf{k}-\omega_{\mathbf{h}}(a)\right)} & \quad+\frac{(-1)^{\lceil\mathbf{k}\rceil+\omega_{\mathbf{h}}(a)}}{2 \pi i(\log M)^{\lceil\mathbf{k}\rceil+\omega_{\mathbf{h}}(a)}} \int_{(-1 / 2)} Z_{9}^{\left(\lceil\mathbf{k}\rceil+\omega_{\mathbf{h}}(a)\right)}(s) M^{s} d s
\end{aligned}
$$

by Lemma 5.10, We will show the bound

$$
Z_{9}^{\left(\lceil\mathbf{k}\rceil+\omega_{\mathbf{h}}(a)\right)}(s) \ll|s+1|^{\mathbf{k}-\lceil\mathbf{k}\rceil-1}
$$

for $s$ close to -1 , which will yield (using Lemma 5.8)

$$
\begin{aligned}
\int_{\left(-1+\frac{1}{\log M}\right)} Z_{9}^{\left(\lceil\mathbf{k}\rceil+\omega_{\mathbf{h}}(a)\right)}(s) M^{s} d s & \ll \frac{1}{M}\left|\int_{-\infty}^{\infty} Z_{9}^{\left(\lceil\mathbf{k}\rceil+\omega_{\mathbf{h}}(a)\right)}\left(-1+\frac{1}{\log M}+i t\right) M^{i t} d t\right| \\
& =\frac{1}{M}\left|\int_{-2}^{2} Z_{9}^{\left(\lceil\mathbf{k}\rceil+\omega_{\mathbf{h}}(a)\right)}\left(-1+\frac{1}{\log M}+i t\right) M^{i t} d t+O(1)\right| \\
& \ll \frac{1}{M}\left(\int_{-2}^{2}\left(\frac{1}{\log M}+|t|\right)^{\mathbf{k}-\lceil\mathbf{k}\rceil-1} d t+O(1)\right) \\
& \ll \frac{1}{M}\left(\int_{0}^{\frac{1}{\log M}}(\log M)^{1-\mathbf{k}+\lceil\mathbf{k}\rceil}+\int_{\frac{1}{\log M}}^{2} t^{\mathbf{k}-\lceil\mathbf{k}\rceil-1} d t+O(1)\right) \\
& \ll \frac{(\log M)^{\lceil\mathbf{k}\rceil-\mathbf{k}}+1}{M} \ll \frac{(\log M)^{\lceil\mathbf{k}\rceil-\mathbf{k}}}{M},
\end{aligned}
$$

from which we will conclude using (17) that

$$
\begin{aligned}
\frac{1}{2 \pi i} \int_{(-1 / 2)} Z(s) M^{s} d s & =\frac{c}{M} \frac{(\log M)^{1-\mathbf{k}-\omega_{\mathbf{h}}(a)}}{\Gamma\left(2-\mathbf{k}-\omega_{\mathbf{h}}(a)\right)}\left(1+O\left(\frac{1}{\log M}\right)\right) \\
& =-\mu_{\mathbf{k}}(a, M)\left(1+O\left(\frac{1}{\log M}\right)\right)
\end{aligned}
$$

achieving the proof. Let us now show that (18) holds. By Lemma 5.6, the function

$$
Z_{10}(s):=(s+1)^{2-\mathbf{k}-\omega_{\mathbf{h}}(a)} Z(s)
$$


as well as its derivatives are locally bounded around $s=-1$. Moreover, applying Lemma 5.4 gives the bound

$$
Z_{10}(s)=Z_{10}(-1)+O(|s+1|) .
$$

Now we use Leibniz's formula:

$$
\begin{aligned}
Z^{\left(\lceil\mathbf{k}\rceil+\omega_{\mathbf{h}}(a)\right)}(s) & =\left((s+1)^{\mathbf{k}+\omega_{\mathbf{h}}(a)-2} Z_{10}(s)\right)^{\left(\lceil\mathbf{k}\rceil+\omega_{\mathbf{h}}(a)\right)} \\
& =\sum_{i=0}^{\lceil\mathbf{k}\rceil+\omega_{\mathbf{h}}(a)}\left(\begin{array}{c}
\lceil\mathbf{k}\rceil+\omega_{\mathbf{h}}(a) \\
i
\end{array}\right)\left((s+1)^{\mathbf{k}+\omega_{\mathbf{h}}(a)-2}\right)^{(i)} Z_{10}^{\left(\lceil\mathbf{k}\rceil+\omega_{\mathbf{h}}(a)-i\right)}(s) \\
& =\left((s+1)^{\mathbf{k}+\omega_{\mathbf{h}}(a)-2}\right)^{\left(\lceil\mathbf{k}\rceil+\omega_{\mathbf{h}}(a)\right)} Z_{10}(s)+O\left(|s+1|^{\mathbf{k}-\lceil\mathbf{k}\rceil-1}\right) \\
& =\left((s+1)^{\mathbf{k}+\omega_{\mathbf{h}}(a)-2}\right)^{\left(\lceil\mathbf{k}\rceil+\omega_{\mathbf{h}}(a)\right)} Z_{10}(-1)+O\left(|s+1|^{\mathbf{k}-\lceil\mathbf{k}\rceil-1}\right)
\end{aligned}
$$

by (19), so

$$
\begin{aligned}
Z_{9}^{\left(\lceil\mathbf{k}\rceil+\omega_{\mathbf{h}}(a)\right)}(s) & =Z^{\left(\lceil\mathbf{k}\rceil+\omega_{\mathbf{h}}(a)\right)}(s)-c\left((s+1)^{\mathbf{k}+\omega_{\mathbf{h}}(a)-2}\right)^{\left(\lceil\mathbf{k}\rceil+\omega_{\mathbf{h}}(a)\right)} \\
& =\left(Z_{10}(-1)-c\right)\left((s+1)^{\mathbf{k}+\omega_{\mathbf{h}}(a)-2}\right)^{\left(\lceil\mathbf{k}\rceil+\omega_{\mathbf{h}}(a)\right)}+O\left(|s+1|^{\mathbf{k}-\lceil\mathbf{k}\rceil-1}\right) \\
& =O\left(|s+1|^{\mathbf{k}-\lceil\mathbf{k}\rceil-1}\right)
\end{aligned}
$$

since $c=Z_{10}(-1)$.

Lemma 5.12. Assume Hypothesis 3.3. Let $y \geq 1$ be a real number. Then,

$$
\frac{1}{2 \pi i} \int_{(-1 / 2)} \mathfrak{S}_{2}(s) \zeta(s+1) \zeta(s+2)^{1-\mathbf{k}} Z_{5}(s) y^{s} \frac{d s}{s} \ll_{\epsilon} y^{-1+\epsilon} .
$$

Proof. Define

$$
Z_{\mathcal{A}}(s):=\mathfrak{S}_{2}(s) \zeta(s+1) \zeta(s+2)^{1-\mathbf{k}} Z_{5}(s) .
$$

The goal is to bound the integral

$$
\frac{1}{2 \pi i} \int_{(-1 / 2)} Z_{\mathcal{A}}(s) y^{s} \frac{d s}{s}=\frac{1}{2 \pi i} \int_{(-1+\epsilon)} Z_{\mathcal{A}}(s) y^{s} \frac{d s}{s} .
$$

We will first show that this integral is $\ll_{\epsilon} y^{-1 / 2+\epsilon}$ using complex analysis, and then we will see how to improve this bound to $\ll_{\epsilon} y^{-1+\epsilon}$ by elementary means. In the region $-1+\epsilon<\sigma$, we have the bound

$$
|Z(\sigma+i t)| \ll_{\epsilon}|\zeta(\sigma+1+i t)| \ll_{\epsilon}(|t|+2)^{\mu(\sigma+1)+\epsilon},
$$


where $\mu(\sigma+1)$ is defined as in Lemma 5.7. Thus we get the bounds

$$
\begin{gathered}
\int_{-1+\epsilon-i T}^{-1+\epsilon+i T} Z(s) y^{s} \frac{d s}{s} \ll_{\epsilon} \frac{T^{1 / 2}}{y^{1-\epsilon}} \\
\int_{-1+\epsilon \pm i T}^{\epsilon \pm i T} Z(s) y^{s} \frac{d s}{s} \ll_{\epsilon}(T y)^{\epsilon}\left(\frac{1}{T^{5 / 6} y^{1 / 2}}+\frac{1}{T^{1 / 2} y}+\frac{1}{T}+\frac{1}{T^{5 / 6} y^{1 / 2}}\right) .
\end{gathered}
$$

The last integral we need to bound is

$$
\begin{aligned}
\frac{1}{2 \pi i} \int_{\Re s=\epsilon,|\Im s|>T} Z(s) y^{s} \frac{d s}{s} & =\sum_{n} \frac{\mathbf{f}_{a}(n)}{n \gamma(n)} \frac{1}{2 \pi i} \int_{\Re s=\epsilon,|\Im s|>T}\left(\frac{y}{n}\right)^{s} \frac{d s}{s} \\
& \ll y^{\epsilon} \sum_{n} \frac{\mathbf{f}_{a}(n)}{n \gamma(n)} \frac{1}{n^{\epsilon}(1+T|\log (y / n)|)}
\end{aligned}
$$

by the effective version of Perron's formula (see Théorème II.2.3 of [17]). The last sum is

$$
\begin{aligned}
& \ll \frac{y^{\epsilon}}{\sqrt{T}} \sum_{n \leq y\left(1-\frac{1}{\sqrt{T}}\right)} \frac{\mathbf{f}_{a}(n)}{n \gamma(n)}+\sum_{y\left(1-\frac{1}{\sqrt{T}}\right) \leq n \leq y\left(1+\frac{1}{\sqrt{T}}\right)} \frac{\mathbf{f}_{a}(n)}{n \gamma(n)}+\frac{y^{\epsilon}}{\sqrt{T}} \sum_{n \geq y\left(1+\frac{1}{\sqrt{T}}\right)} \frac{\mathbf{f}_{a}(n)}{n \gamma(n)} \frac{1}{n^{\epsilon}} \\
& \ll \frac{y^{\epsilon}}{\sqrt{T}} \log y+\frac{1}{\sqrt{T}} \ll_{\epsilon} \frac{y^{\epsilon}}{\sqrt{T}} \log y
\end{aligned}
$$

by Lemma 5.2. Taking $T=y$ yields that the left hand side of $(20)$ is $\ll_{\epsilon} y^{-1 / 2+\epsilon}$. We now proceed to show this bound can be improved to $\ll_{\epsilon} y^{-1+\epsilon}$. The function $Z_{\mathcal{A}}(s) y^{s} / s$ has a double pole at $s=0$ with residue equal to $C_{1} \log y+C_{2}$, where $C_{1}$ and $C_{2}$ are real numbers independent of $y$. By the residue theorem and Mellin inversion,

$$
\begin{aligned}
\frac{1}{2 \pi i} \int_{(-1 / 2)} Z_{\mathcal{A}}(s) y^{s} \frac{d s}{s} & =-C_{1} \log y-C_{2}+\frac{1}{2 \pi i} \int_{(1)} Z_{\mathcal{A}}(s) y^{s} \frac{d s}{s} \\
& =\sum_{n \leq y} \frac{\mathbf{f}_{a}(n)}{n \gamma(n)}-C_{1} \log y-C_{2} .
\end{aligned}
$$

Let us give an elementary estimate for the sum appearing on the right hand side of (21). Define

$$
\nu(n):=\prod_{p \mid n} \frac{1-\mathbf{h}(p)}{p-1} .
$$

Using the convolution identity

$$
\frac{1}{\gamma(n)}=\sum_{\substack{r s=n \\ 27}} \mu^{2}(s) \nu(s)
$$


we compute

$$
\begin{aligned}
& \sum_{n \leq y} \frac{\mathbf{f}_{a}(n)}{n \gamma(n)}=\sum_{s \leq y} \frac{\mu^{2}(s) \nu(s)}{s} \sum_{r \leq y / s} \frac{\mathbf{f}_{a}(r s)}{r}=\sum_{s \leq y} \frac{\mu^{2}(s) \nu(s)}{s} \sum_{(a, s)|d| a} \mathbf{f}_{a}(d) \sum_{\substack{r \leq y / s: \\
(a, r s)=d}} \frac{1}{r} \\
& =\sum_{s \leq y} \frac{\mu^{2}(s) \nu(s)}{s} \sum_{(a, s)|d| a} \mathbf{f}_{a}(d) \sum_{\substack{r \leq y / s: \\
\frac{d}{(d, s)} \\
(a, r s)=d}} \frac{1}{r} \\
& =\sum_{s \leq y} \frac{\mu^{2}(s) \nu(s)}{s} \sum_{(a, s)|d| a} \mathbf{f}_{a}(d) \frac{(d, s)}{d} \sum_{\substack{l \leq \frac{y(d, s)}{d s}: \\
(a / d, l s /(d, s))=1}} \frac{1}{l} \\
& =\sum_{s \leq y} \frac{\mu^{2}(s) \nu(s)}{s} \sum_{\substack{(a, s)|d| a: \\
(a / d, s /(d, s))=1}} \mathbf{f}_{a}(d) \frac{(d, s)}{d} \sum_{\substack{l \leq \frac{y(d, s)}{d s}: \\
(l, a / d)=1}} \frac{1}{l} \\
& =\sum_{s \leq y} \frac{\mu^{2}(s) \nu(s)}{s} \sum_{\substack{(a, s)|d| a: \\
(a / d, s /(d, s))=1}} \mathbf{f}_{a}(d) \frac{(d, s)}{d} \frac{\phi(a / d)}{a / d}\left(\log \left(\frac{y(d, s)}{d s}\right)+\gamma\right. \\
& \left.+\sum_{p \mid a / d} \frac{\log p}{p-1}+O\left(\frac{d s}{y(d, s)}\right)\right) \text {. }
\end{aligned}
$$

Using the bound $\nu(n) \ll_{\epsilon} n^{-1+\epsilon}$, which is deduced from Hypothesis 3.3. we get that the error terms sum to $O_{a, \epsilon}\left(y^{-1+\epsilon}\right)$. Moreover, we can extend the sum over $s \leq y$ to all integers, at the cost of the error term $O_{a, \epsilon}\left(y^{-1+\epsilon}\right)$. Having done this, (22) becomes

$$
\sum_{n \leq y} \frac{\mathbf{f}_{a}(n)}{n \gamma(n)}=\tilde{C}_{1} \log y+\tilde{C}_{2}+O_{a, \epsilon}\left(y^{-1+\epsilon}\right)
$$

where $\tilde{C}_{1}$ and $\tilde{C}_{2}$ are real numbers which do not depend on $y$. Substituting (23) into (21) and using our previous bound, we get

$$
\left(\tilde{C}_{1}-C_{1}\right) \log y+\tilde{C}_{2}-C_{2}+O_{a, \epsilon}\left(y^{-1+\epsilon}\right)=\frac{1}{2 \pi i} \int_{(-1 / 2)} Z_{\mathcal{A}}(s) y^{s} \frac{d s}{s}=O_{a, \epsilon}\left(y^{-1 / 2+\epsilon}\right)
$$

which of course implies that $\tilde{C}_{1}=C_{1}$ and $\tilde{C}_{2}=C_{2}$ since these numbers do not depend on $y$. We conclude from (21) and (23) that (20) holds. 


\subsubsection{Proof of Proposition 5.1.}

Proof of Proposition 5.1. First we use Lemma 5.3 to write

$$
\begin{aligned}
S_{5}: & =\sum_{1 \leq r \leq \mathbf{R}} \frac{\mathbf{f}_{a}(r)}{r \gamma(r)}\left(1-\frac{r}{\mathbf{R}}\right)-\sum_{1 \leq r \leq M} \frac{\mathbf{f}_{a}(r)}{r \gamma(r)}\left(1-\frac{r}{M}\right)-\sum_{\frac{x}{R}<q \leq \frac{x}{M}} \frac{\mathbf{f}_{a}(q)}{q \gamma(q)} \\
& =\frac{1}{2 \pi i} \int_{(1)} \mathfrak{S}_{2}(s) \zeta(s+1) \zeta(s+2)^{1-\mathbf{k}} Z_{5}(s)\left(\frac{\mathbf{R}^{s}-M^{s}}{s+1}+\left(\frac{x}{\mathbf{R}}\right)^{s}-\left(\frac{x}{M}\right)^{s}\right) \frac{d s}{s} .
\end{aligned}
$$

Writing

$$
\psi(s):=\frac{\mathbf{R}^{s}-M^{s}}{s+1}+\left(\frac{x}{\mathbf{R}}\right)^{s}-\left(\frac{x}{M}\right)^{s},
$$

it is trivial that $\psi(0)=0$. Using Taylor series, we have for $s$ close to 0 that

$$
\psi(s)=(1+O(s))\left(s \log (\mathbf{R} / M)+O\left(s^{2}\right)\right)+s \log (x / \mathbf{R})-s \log (x / M)+O\left(s^{2}\right),
$$

which means that $\psi$ has a double zero at $s=0$. Thus,

$$
\mathfrak{S}_{2}(s) \zeta(s+1) \zeta(s+2)^{1-\mathbf{k}} Z_{5}(s) \frac{\psi(s)}{s}
$$

is holomorphic at $s=0$. Using this fact,

$$
\begin{aligned}
S_{5} & =\frac{1}{2 \pi i} \int_{(-1 / 2)} \mathfrak{S}_{2}(s) \zeta(s+1) \zeta(s+2)^{1-\mathbf{k}} Z_{5}(s) \psi(s) \frac{d s}{s} \\
& =\frac{1}{2 \pi i} \int_{(-1 / 2)} \mathfrak{S}_{2}(s) \zeta(s+1) \zeta(s+2)^{1-\mathbf{k}} Z_{5}(s)\left(\mathbf{R}^{s}-M^{s}\right) \frac{d s}{s(s+1)}+O_{\epsilon}\left(\left(\frac{\mathbf{R}}{x}\right)^{1-\epsilon}\right)
\end{aligned}
$$

by Lemma 5.12. We conclude using propositions 5.9 and 5.11 that

$$
\begin{aligned}
S_{5}= & \frac{\mu_{\mathbf{k}}(a, M)}{M}\left(1+O\left(\frac{\log \log M}{\log M}\right)\right)+O_{A}\left(\frac{1}{M \log ^{A} M}\right) \\
& \quad-\frac{\mu_{\mathbf{k}}(a, \mathbf{R})}{\mathbf{R}}\left(1+O\left(\frac{\log \log \mathbf{R}}{\log \mathbf{R}}\right)\right)+O_{A}\left(\frac{1}{\mathbf{R} \log ^{A} \mathbf{R}}\right)+O_{\epsilon}\left(\left(\frac{\mathbf{R}}{x}\right)^{1-\epsilon}\right) \\
= & \frac{\mu_{\mathbf{k}}(a, M)}{M}\left(1+O\left(\frac{\log \log M}{\log M}\right)\right)+O_{A}\left(\frac{1}{M \log ^{A} M}\right)
\end{aligned}
$$

since $M(x)^{1+\delta} \leq \mathbf{L}(x)^{1+\delta} \leq \mathbf{R}(x) \leq \sqrt{x}$. 
5.2. Proofs of theorems 4.1 and $4.1^{*}$. We first define the following counting function, which will come in handy for the proofs of this section:

$$
\mathcal{A}^{*}(x ; q, a):=\sum_{\substack{|a|<n \leq x \\ n \equiv a \bmod q}} \mathbf{a}(n) .
$$

Proof of Theorem 4.1. Let $1 \leq M(x) \leq \mathbf{L}(x)$ and let $\mathbf{R}=\mathbf{R}(x)$ be as in Hypothesis 3.1. We decompose the sum (8) as follows:

$$
\begin{gathered}
\sum_{q \leq \frac{x}{M}}\left(\mathcal{A}(x ; q, a)-\mathbf{a}(a)-\frac{\mathbf{f}_{a}(q)}{q \gamma(q)} \mathcal{A}(x)\right)=\sum_{q \leq \frac{x}{M}}\left(\mathcal{A}^{*}(x ; q, a)-\frac{\mathbf{f}_{a}(q)}{q \gamma(q)} \mathcal{A}(x)\right)+O(1) \\
=\sum_{\frac{x}{\mathrm{R}}<q \leq x} \mathcal{A}^{*}(x ; q, a)-\sum_{\frac{x}{M}<q \leq x} \mathcal{A}^{*}(x ; q, a)-\mathcal{A}(x) \sum_{\frac{x}{\mathrm{R}}<q \leq \frac{x}{M}} \frac{\mathbf{f}_{a}(q)}{q \gamma(q)} \\
+\sum_{q \leq \frac{x}{\mathrm{R}}}\left(\mathcal{A}^{*}(x ; q, a)-\frac{\mathbf{f}_{a}(q)}{q \gamma(q)} \mathcal{A}(x)\right)+O(1) \\
=S_{1}-S_{2}-S_{3}+S_{4}+O(1) .
\end{gathered}
$$

Hypothesis 3.4 implies the bound

$$
S_{4} \ll \frac{\mathcal{A}(x)}{M(x)^{1+\delta}} .
$$

To evaluate the sums $S_{1}$ and $S_{2}$ we use the Hooley-Montgomery divisor switching technique (see [9]). Setting $n=a+q r$, we have for positive $a$ that

$$
\begin{aligned}
& S_{2}=\sum_{\frac{x}{M}<q \leq x} \sum_{\substack{|a|<n \leq x \\
n \equiv a \bmod q}} \mathbf{a}(n)=\sum_{\substack{1 \leq r<(x-a) \frac{M}{x} \\
a+r \frac{x}{M}<n \leq x \\
n \equiv a \bmod r}} \mathbf{a}(n) \\
& =\sum_{1 \leq r<(x-a) \frac{M}{x}}\left(\mathcal{A}(x ; r, a)-\mathcal{A}\left(a+r \frac{x}{M} ; r, a\right)\right) .
\end{aligned}
$$

Using Hypothesis 3.1, we see that there exists $\delta>0$ such that

$$
\begin{aligned}
S_{2} & =\sum_{1 \leq r<(x-a) \frac{M}{x}} \frac{\mathbf{f}_{a}(r)}{r \gamma(r)}\left(\mathcal{A}(x)-\mathcal{A}\left(a+r \frac{x}{M}\right)\right)+O\left(\frac{\mathcal{A}(x)}{\mathbf{L}(x)^{1+2 \delta}}\right) \\
& =\sum_{1 \leq r<(x-a) \frac{M}{x}} \frac{\mathbf{f}_{a}(r)}{r \gamma(r)}\left(\mathcal{A}(x)-\mathcal{A}\left(\frac{r}{M} x\right)\right)+O\left(\frac{\mathcal{A}(x)}{\mathbf{L}(x)^{1+\delta}}\right) \\
& =\mathcal{A}(x) \sum_{1 \leq r<(x-a) \frac{M}{x}} \frac{\mathbf{f}_{a}(r)}{r \gamma(r)}\left(1-\frac{\mathcal{A}\left(\frac{r}{M} x\right)}{\mathcal{A}(x)}\right)+O\left(\frac{\mathcal{A}(x)}{\mathbf{L}(x)^{1+\delta}}\right)
\end{aligned}
$$


by hypotheses 3.2 and Lemma 5.2. Now, if $a$ were negative, we would have to add an error term of size $\ll \frac{\mathcal{A}(x)}{\mathbf{L}(x)^{1+\delta}}$ to (26) (by Hypothesis 3.2$)$, which would yield the same error term in (27). Using Hypothesis 3.2 again, (27) becomes

$$
=\mathcal{A}(x) \sum_{1 \leq r<(x-a) \frac{M}{x}} \frac{\mathbf{f}_{a}(r)}{r \gamma(r)}\left(1-\frac{r}{M}\right)+O\left(\frac{\mathcal{A}(x)}{\mathbf{L}(x)^{1+\delta}}\right) .
$$

If $M$ is an integer, then the $M$-th term of the sum is $\frac{\mathrm{f}_{a}(r)}{r \gamma(r)}\left(1-\frac{M}{M}\right)=0$. If not, the bound $\frac{\mathrm{f}_{a}(r)}{r \gamma(r)} \ll_{\epsilon} \frac{1}{\phi(r)}$ (see Lemma 5.2) implies that this last term is $\ll \mathcal{A}(x) \frac{\log \log M}{M^{2}}$. Thus,

$$
S_{2}=\mathcal{A}(x) \sum_{1 \leq r \leq M} \frac{\mathbf{f}_{a}(r)}{r \gamma(r)}\left(1-\frac{r}{M}\right)+O\left(\frac{\mathcal{A}(x)}{M^{1+\delta}}\right)
$$

since $M(x) \leq \mathbf{L}(x)$. A similar calculation shows that

$$
S_{1}=\mathcal{A}(x) \sum_{1 \leq r \leq \mathbf{R}(x)} \frac{\mathbf{f}_{a}(r)}{r \gamma(r)}\left(1-\frac{r}{\mathbf{R}(x)}\right)+O\left(\frac{\mathcal{A}(x)}{\mathbf{L}(x)^{1+\delta}}\right) .
$$

Grouping terms, (25) becomes

$$
\begin{gathered}
\sum_{q \leq \frac{x}{M}}\left(\mathcal{A}(x ; q, a)-\mathbf{a}(a)-\frac{\mathbf{f}_{a}(q)}{q \gamma(q)} \mathcal{A}(x)\right)=S_{1}-S_{2}-S_{3}+S_{4}+O(1) \\
=\mathcal{A}(x)\left(\sum_{1 \leq r \leq \mathbf{R}} \frac{\mathbf{f}_{a}(r)}{r \gamma(r)}\left(1-\frac{r}{\mathbf{R}}\right)-\sum_{1 \leq r \leq M} \frac{\mathbf{f}_{a}(r)}{r \gamma(r)}\left(1-\frac{r}{M}\right)-\sum_{\frac{x}{\mathrm{R}}<q \leq \frac{x}{M}} \frac{\mathbf{f}_{a}(q)}{q \gamma(q)}\right) \\
+O\left(\frac{\mathcal{A}(x)}{M^{1+\delta}}\right),
\end{gathered}
$$

which combined with Proposition 5.1 gives

$$
=\frac{\mathcal{A}(x)}{M} \mu_{\mathbf{k}}(a, M)\left(1+O\left(\frac{\log \log M}{\log M}\right)\right)+O_{A}\left(\frac{\mathcal{A}(x)}{M \log ^{A} M}\right),
$$

that is

$$
\begin{aligned}
\sum_{q \leq \frac{x}{M}}(\mathcal{A}(x ; q, a)-\mathbf{a}(a)- & \left.\frac{\mathbf{f}_{a}(q)}{q \gamma(q)} \mathcal{A}(x)\right) \\
& =\frac{\mathcal{A}(x)}{M}\left(\mu_{\mathbf{k}}(a, M)\left(1+O\left(\frac{\log \log M}{\log M}\right)\right)+O_{A}\left(\frac{1}{\log ^{A} M}\right)\right) .
\end{aligned}
$$


Proof of Theorem $[4.1]^{*}$. Let $1 \leq M(x) \leq \mathbf{L}(x)$ and let $\mathbf{R}=\mathbf{R}(x)$ be as in Hypothesis 3.4. We decompose the sum (9) as follows:

$$
\begin{aligned}
\sum_{\frac{x}{2 M}<q \leq \frac{x}{M}} & \left(\mathcal{A}(x ; q, a)-\mathbf{a}(a)-\frac{\mathbf{f}_{a}(q)}{q \gamma(q)} \mathcal{A}(x)\right)=\sum_{\frac{x}{2 M}<q \leq \frac{x}{M}}\left(\mathcal{A}^{*}(x ; q, a)-\frac{\mathbf{f}_{a}(q)}{q \gamma(q)} \mathcal{A}(x)\right)+O(1) \\
& =\sum_{\frac{x}{2 M}<q \leq x} \mathcal{A}^{*}(x ; q, a)-\sum_{\frac{x}{M}<q \leq x} \mathcal{A}^{*}(x ; q, a)-\mathcal{A}(x) \sum_{\frac{x}{2 M}<q \leq \frac{x}{M}} \frac{\mathbf{f}_{a}(q)}{q \gamma(q)}+O(1) \\
& =S_{1}-S_{2}-S_{3}+O(1) .
\end{aligned}
$$

Arguing as in the proof of Theorem 4.1, we set $n=a+q r$ to get that for positive $a$,

$$
\begin{aligned}
S_{2} & =\sum_{1 \leq r<(x-a) \frac{M}{x}}\left(\mathcal{A}(x ; r, a)-\mathcal{A}\left(a+r \frac{x}{M} ; r, a\right)\right) \\
& =\mathcal{A}(x) \sum_{1 \leq r<(x-a) \frac{M}{x}} \frac{\mathbf{f}_{a}(r)}{r \gamma(r)}\left(1-\frac{\mathcal{A}\left(\frac{r}{M} x\right)}{\mathcal{A}(x)}\right)+O\left(\frac{\mathcal{A}(x)}{\mathbf{L}(x)^{1+\delta}}\right) \\
& =\mathcal{A}(x) \sum_{1 \leq r \leq M} \frac{\mathbf{f}_{a}(r)}{r \gamma(r)}\left(1-\frac{r}{M}\right)+O\left(\frac{\mathcal{A}(x)}{M^{1+\delta}}\right)
\end{aligned}
$$

by Hypotheses $\left[3.11^{*}, 3.2\right.$ and Lemma 5.2. Now, if $a$ were negative, we would have to add a negligible contribution. Thus, (28) becomes

$$
\begin{gathered}
\sum_{\frac{x}{2 M}<q \leq \frac{x}{M}}\left(\mathcal{A}(x ; q, a)-\mathbf{a}(a)-\frac{\mathbf{f}_{a}(q)}{q \gamma(q)} \mathcal{A}(x)\right)=\mathcal{A}(x)\left(\sum_{1 \leq r \leq 2 M} \frac{\mathbf{f}_{a}(r)}{r \gamma(r)}\left(1-\frac{r}{2 M}\right)\right. \\
\left.-\sum_{1 \leq r \leq M} \frac{\mathbf{f}_{a}(r)}{r \gamma(r)}\left(1-\frac{r}{M}\right)-\sum_{\frac{x}{2 M}<q \leq \frac{x}{M}} \frac{\mathbf{f}_{a}(q)}{q \gamma(q)}\right)+O\left(\frac{\mathcal{A}(x)}{M^{1+\delta}}\right) .
\end{gathered}
$$

Going through the proof of Proposition 5.1, we see that this is

$$
\begin{aligned}
=\frac{\mathcal{A}(x)}{M} \mu_{\mathbf{k}}(a, M)\left(1+O\left(\frac{\log \log M}{\log M}\right)\right)-\frac{\mathcal{A}(x)}{2 M} \mu_{\mathbf{k}}(a, 2 M)\left(1+O\left(\frac{\log \log M}{\log M}\right)\right) \\
+O_{A}\left(\frac{\mathcal{A}(x)}{M \log ^{A} M}\right)
\end{aligned}
$$


that is

$$
\begin{aligned}
\sum_{\frac{x}{2 M}<q \leq \frac{x}{M}}(\mathcal{A}(x ; q, a)-\mathbf{a}(a) & \left.-\frac{\mathbf{f}_{a}(q)}{q \gamma(q)} \mathcal{A}(x)\right) \\
= & \frac{\mathcal{A}(x)}{2 M}\left(\mu_{\mathbf{k}}(a, M)\left(1+O\left(\frac{\log \log M}{\log M}\right)\right)+O_{A}\left(\frac{1}{\log ^{A} M}\right)\right),
\end{aligned}
$$

since by the definition of $\mu_{\mathbf{k}}(a, M)$,

$$
2 \mu_{\mathbf{k}}(a, M)-\mu_{\mathbf{k}}(a, 2 M)=\mu_{\mathbf{k}}(a, M)\left(1+O\left(\frac{1}{\log M}\right)\right) .
$$

\section{Further Proofs}

In this section we prove the results of Section 2 ,

Proof of Theorem 2.2. Put

$$
a(n):=\Lambda(n)
$$

which gives $\mathcal{A}(x)=\psi(x)$ and $\mathcal{A}(x ; q, a)=\psi(x ; q, a)$. Define

$$
\mathbf{f}_{a}(q):= \begin{cases}1 & \text { if }(a, q)=1 \\ 0 & \text { otherwise }\end{cases}
$$

and $\gamma(q):=\frac{\phi(q)}{q}$. Define also the multiplicative function $\mathbf{h}(d)$ by $\mathbf{h}(1)=1$, and $\mathbf{h}(d)=0$ for $d>1$. The prime number theorem in arithmetic progressions gives the asymptotic

$$
\mathcal{A}(x ; q, a) \sim \frac{\mathbf{f}_{a}(q)}{q \gamma(q)} \mathcal{A}(x),
$$

for any fixed $a$ and $q$ such that $(a, q)=1$. Now let us show that the hypotheses of Section 3.2 hold. Fix $A>0$ and put $\mathbf{L}(x):=(\log x)^{A}, \mathbf{R}(x):=x^{1 / 2}(\log x)^{-B(A)}$, where $B(A):=A+5$. Hypothesis 3.1 is the Bombieri-Vinogradov theorem. Hypothesis 3.2 follows from the prime number theorem. As $\mathbf{h}(p)=\mathbf{k}=0$, Hypothesis 3.3 is trivial. Hypothesis 3.4 follows from Theorem 9 of [3].

We now compute $\mu_{\mathbf{k}}(a, M)$. As $\mathbf{h}\left(p^{e}\right)=0$, we have $\omega_{\mathbf{h}}(a)=\omega(a)$, the number of prime factors of $a$. Thus, Remark 4.4 gives 


$$
\mu_{0}(a, M)= \begin{cases}-\frac{1}{2} \log M & \text { if } a= \pm 1 \\ -\frac{1}{2}\left(1-\frac{1}{p}\right) \log p & \text { if } a= \pm p^{e} \\ 0 & \text { if } \omega(a) \geq 2\end{cases}
$$

so an application of Theorem 4.1 gives the result with a weaker error term. A better version of Proposition 5.1 follows from Huxley's subconvexity result [10], yielding the stated error term (see [7] for a more precise proof).

Proof of Theorem 2.3. Let $Q(x, y):=\alpha x^{2}+\beta x y+\gamma y^{2}$ be a binary quadratic form, where $\alpha, \beta$ and $\gamma$ are integers such that $\alpha>0,(\alpha, \beta, \gamma)=1$ and $d:=\beta^{2}-4 \alpha \gamma<0$ (so $Q(x, y)$ is positive definite). Note that the set of $d$ for which $d \equiv 1,5,9,12,13 \bmod 16$ includes a large subset of all fundamental discriminants. The set of bad primes is $\mathcal{S}:=\{p: p \mid 2 d\}$ in this case. Since $\mathcal{S} \neq \emptyset$, we will need to modify the proof of Theorem 4.1. We define

$$
\chi_{d}:=\left(\frac{4 d}{\cdot}\right)
$$

Note that for $(n, 2 d)=1$, we have the equalities

$$
r_{d}(n)=\sum_{m \mid n} \chi_{d}(m)=\prod_{\substack{p^{k} \| n: \\
\chi_{d}(p)=1}}(k+1) \prod_{\begin{array}{r}
p^{k} \| n: \\
\chi_{d}(p)=-1 \\
k \text { odd }
\end{array}} 0
$$

An intuitive argument suggests that

$$
\mathcal{A}(x ; q, a) \sim \frac{R_{a}(q)}{q^{2}} \mathcal{A}(x)
$$

where

$$
R_{a}(q):=\#\{1 \leq x, y \leq q: Q(x, y) \equiv a \bmod q\}
$$

As this is a classical result, we leave its proof, as well as several other classical facts about binary quadratic forms, to Appendix A. The function

$$
\mathbf{g}_{a}(q):=\frac{R_{a}(q)}{q^{2}}
$$


is actually multiplicative (see Lemma A.1), and Lemma A.3 shows that for $p \nmid 2 d, \mathbf{g}_{a}$ is given as in (5) with

$$
\mathbf{h}\left(p^{e}\right):= \begin{cases}1+e\left(1-\frac{1}{p}\right) & \text { if } \chi_{d}(p)=1 \\ \frac{1}{p} & \text { if } \chi_{d}(p)=-1 \text { and } 2 \nmid e \\ 1 & \text { if } \chi_{d}(p)=-1 \text { and } 2 \mid e,\end{cases}
$$

and for $p \mid 2 d, R_{a}\left(p^{e}\right)$ is given as in (48) and (49). Since we are looking at large moduli, we need to use a result of Plaksin (Lemma 8 of [16]), which asserts that

$$
\mathcal{A}(x ; q, a)=\mathbf{g}_{a}(q) \mathcal{A}(x)+E(x, q)
$$

where $E(x, q) \ll_{a, \epsilon}(x / q)^{\frac{3}{4}+\epsilon}$ if $q \leq x^{\frac{1}{3}}$, and $E(x, q) \ll_{a, \epsilon} x^{\frac{2}{3}+\epsilon} q^{-\frac{1}{2}}$ if $x^{\frac{1}{3}}<q \leq x^{\frac{2}{3}}$. Summing (31) over $q \leq x^{\frac{1}{2}}$, we get that the hypotheses 3.1 and 3.4 hold with $\mathbf{R}(x):=x^{\frac{1}{2}}$ and $\mathbf{L}(x):=x^{\lambda}$, provided $\lambda<\frac{1}{12}$. (Note that in the case $\beta=0$, we can take the wider range $\lambda<\frac{1}{8}$, using Lemma 20 of [15].) Hypothesis 3.2 follows from Gauss' estimate:

$$
\mathcal{A}(x)=A_{Q} x+O\left(x^{\frac{1}{2}}\right)
$$

where $A_{Q}$ is the area of the region $\left\{(x, y) \in \mathbb{R}_{\geq 0}^{2}: Q(x, y) \leq 1\right\}$. Let us turn to Hypothesis 3.3. For $p \nmid 2 d$,

$$
\mathbf{h}(p)= \begin{cases}2-\frac{1}{p} & \text { if } \chi_{d}(p)=1 \\ \frac{1}{p} & \text { if } \chi_{d}(p)=-1\end{cases}
$$

so we set $\mathbf{k}:=1$ and

$$
\sum_{p \notin \mathcal{S}} \frac{\mathbf{h}(p)-\mathbf{k}}{p}=\sum_{p \nmid 2 d} \frac{\chi_{-d}(p)}{p}+O(1)<\infty
$$

by the prime number theorem for $\psi\left(x, \chi_{-d}\right)$ (see [4]). Moreover,

$$
\begin{aligned}
\sum_{p \notin \mathcal{S}} \frac{(\mathbf{h}(p)-\mathbf{k})(\log p)^{n+1}}{p^{1+i t}} & =O(1)+(-1)^{n+1}\left(\frac{L^{\prime}}{L}\right)^{(n)}\left(1+i t, \chi_{-d}\right) \\
& \ll_{d, n}(\log (|t|+2))^{n+2},
\end{aligned}
$$

this last bound following from Cauchy's formula for the derivatives combined with the classical bound for $\frac{L^{\prime}(s, \chi)}{L(s, \chi)}$ in a zero-free region (see Chapter 19 of [4]). As in the proof of Théorème 
II.3.22 of [17], we can deduce from (32) that (setting $\eta:=1 / \log ^{2}(|t|+2)$ )

$$
\begin{aligned}
\sum_{p \notin \mathcal{S}} \frac{\mathbf{h}(p)-\mathbf{k}}{p^{1+i t}}+O(1) & =\log L\left(1+i t, \chi_{-d}\right)=\int_{1+i t+\eta}^{1+i t} \frac{L^{\prime}\left(s, \chi_{-d}\right)}{L\left(s, \chi_{-d}\right)} d s+\log L\left(1+i t+\eta, \chi_{-d}\right) \\
& \ll \eta \log ^{2}(|t|+2)+\log \zeta(1+\eta)=2 \log \log (|t|+2)+O(1) .
\end{aligned}
$$

Having proven hypotheses $3.1,3.2,3.3$ and 3.4, we now proceed to prove an analogue of Theorem 4.1 (since the set $\mathcal{S}$ is non-empty). In the proof of Lemma 5.3, we need to change the definition of $\mathfrak{S}_{2}(s)\left(=\mathfrak{S}_{1}(s)\right)$ to (remember that $(a, 2 d)=1$ )

$$
\begin{aligned}
\mathfrak{S}_{2}(s) & =\left(\left(1-\frac{1}{2^{s+1}}\right)\left(1+\frac{R_{a}(2)}{2^{s+2}}\right)+\frac{R_{a}(4)}{4} \frac{1}{2^{2 s+2}}\right) \prod_{\substack{p \mid d \\
p \neq 2}}\left(1-\frac{1}{p^{s+1}}+\frac{R_{a}(p)}{p^{s+2}}\right) \\
& \times \prod_{\substack{p^{f} \| a \\
f \geq 1 \\
p \notin \mathcal{S}}}\left[\left(1+\frac{\mathbf{h}(p)}{p^{s+1}}+\ldots+\frac{\mathbf{h}\left(p^{f}\right)}{p^{f(s+1)}}\right)\left(1-\frac{1}{p^{s+1}}\right)+\frac{\mathbf{h}\left(p^{f}\right)-\mathbf{h}\left(p^{f+1}\right) / p}{1-1 / p} \frac{1}{p^{(f+1)(s+1)}}\right],
\end{aligned}
$$

(We also need to change the condition on the product defining $Z_{5}(s)$ to $p \nmid 2 a d$ ) so

$$
\begin{aligned}
\mathfrak{S}_{2}(-1) & =\frac{R_{a}(4)}{4} \prod_{\substack{p \mid d \\
p \neq 2}} \frac{R_{a}(p)}{p} \prod_{\substack{p^{f} \| a \\
f \neq 1 \\
p \notin \mathcal{S}}} \frac{\mathbf{h}\left(p^{f}\right)-\mathbf{h}\left(p^{f+1}\right) / p}{1-1 / p} \\
& =\frac{R_{a}(4)}{4} \prod_{\substack{p^{f} \| d \\
p \neq 2}} \frac{R_{a}\left(p^{f}\right)}{p^{f}} \prod_{\substack{p^{f} \| a: \\
\chi_{d}(p)=1}}\left(1-\frac{1}{p}\right)(f+1) \prod_{\substack{p^{f} \| a: \\
\chi_{d}(p)=-1, f \text { even }}}\left(1+\frac{1}{p}\right) \prod_{\substack{p^{f} \| a: \\
\chi_{d}(p)=-1, f \text { odd }}} 0 \\
& =\frac{R_{a}(4 d)}{4 d} \prod_{p \mid a}\left(1-\frac{\chi_{d}(p)}{p}\right) r_{d}(|a|),
\end{aligned}
$$

by (29) and Lemma A.3. We conclude that Theorem 4.1 holds with

$$
\mu_{1}(a, M)=-\frac{R_{a}(4 d)}{4 d} \cdot \frac{r_{d}(|a|)}{2 L\left(1, \chi_{d}\right)},
$$

which gives the result (with a weaker error term) by Dirichlet's class number formula. To get the better error term $O_{\epsilon}\left(\frac{1}{M^{1 / 3-\epsilon}}\right)$, one has to get a better estimate in Proposition 5.1 , To do this, we go back to the proof of Proposition 5.9 and remark that (with the notation introduced there)

$$
Z_{5}(s)=\prod_{p \nmid 2 a d}\left(1-\frac{\chi_{d}(p)}{p^{s+2}}\right)
$$


So

$$
Z(s)=\frac{\mathfrak{S}_{3}(s) \zeta(s+1) L\left(s+2, \chi_{d}\right)}{s(s+1)},
$$

where

$$
\mathfrak{S}_{3}(s):=\mathfrak{S}_{2}(s) \prod_{p \mid 2 a d}\left(1-\frac{\chi_{d}(p)}{p^{s+2}}\right)^{-1} .
$$

Since $Z(s)$ is a meromorphic function on the whole complex plane, we can shift the contour of integration to the left until the line $\Re(s)=-\frac{4}{3}+\epsilon$. A standard residue calculation combined with the convexity bound on $L\left(s, \chi_{d}\right)$ gives

$$
\frac{1}{2 \pi i} \int_{(-1 / 2)} \frac{\mathfrak{S}_{3}(s) \zeta(s+1) L\left(s+2, \chi_{d}\right)}{s(s+1)} M^{s} d s=-\frac{\mu_{1}(a, M)}{M}+O_{\epsilon}\left(\frac{1}{M^{4 / 3-\epsilon}}\right),
$$

from which we conclude the result.

Proof of Theorem 2.4. Set $\mathcal{S}:=\{2\}, \mathbf{k}:=\frac{1}{2}$ and $\mathbf{L}(x):=(\log x)^{\lambda}$ with $\lambda<1 / 5$. We first prove Hypothesis 3.2 using a refinement of a theorem of Landau. We have

$$
\mathcal{A}(x)=C \frac{x}{\sqrt{\log x}}\left(1+O\left(\frac{x}{\log x}\right)\right),
$$

with

$$
C:=\frac{1}{\sqrt{2}} \prod_{p \equiv 3 \bmod 4}\left(1-\frac{1}{p^{2}}\right)^{-\frac{1}{2}} .
$$

(See for instance Exercice 240 of [17]). The distribution of $\mathcal{A}$ in the arithmetic progressions $a \bmod q$ with $(a, q)=1$ is uniform, however a result of the strength of Plaksin's (31) is far from being known. The best result so far for individual values of $q$ (in terms of uniformity in $q$ ) is due to Iwaniec [11, which proved using the semi-linear sieve that if $(a, q)=1$ and $a \equiv 1 \bmod (q, 4)$, then

$$
\mathcal{A}(x ; q, a)=\frac{(2, q)}{(4, q) q \gamma(q)} \mathcal{A}(x)\left(1+O\left(\frac{\log q}{\log x}\right)^{1 / 5}\right),
$$

where

$$
\gamma(q):=\prod_{\substack{p \mid q \\ p \equiv 3 \bmod 4}}\left(1+\frac{1}{p}\right)^{-1} .
$$

An easy computation using the arithmetic properties of $\mathbf{a}(n)$ shows that

$$
\mathcal{A}_{p^{e}}(x)= \begin{cases}\mathcal{A}\left(\frac{x}{p^{e+1}}\right) & \text { if } p \equiv 3 \bmod 4 \text { and } 2 \nmid e \\ \mathcal{A}\left(\frac{x}{p^{e}}\right) & \text { otherwise, }\end{cases}
$$


and more generally,

$$
\mathcal{A}_{d}(x)=\mathcal{A}\left(\frac{\mathbf{h}(d)}{d} x\right)
$$

with

$$
\mathbf{h}\left(p^{e}\right):= \begin{cases}\frac{1}{p} & \text { if } p \equiv 3 \bmod 4 \text { and } 2 \nmid e \\ 1 & \text { otherwise. }\end{cases}
$$

This confirms that our choice of $\mathbf{k}=\frac{1}{2}$ was good, and Hypothesis 3.3 follows as in the proof of Theorem 2.3. Moreover, (34) can be extended to $(a, q)=d$ for any fixed odd integer $d>1$, by using the identity $\mathcal{A}(x ; q, a)=\mathcal{A}\left(\frac{\mathbf{h}(d)}{d} x ; \frac{q}{d}, \frac{a}{d}\right)$, hence Hypothesis $3.1^{*}$ holds. As we have shown every hypothesis, we turn to the calculation of the average $\mu_{\frac{1}{2}}(a, M)$ (which is never zero since $\mathbf{k} \notin \mathbb{Z})$. We need to modify the definition of $\mathfrak{S}_{2}(s)$, changing the local factor at $p=2$ to

$$
\left(1-\frac{1}{2^{s+2}}\right)^{1 / 2}\left(1-\frac{1}{2^{2 s+2}}+\frac{1}{2^{2 s+3}}\right) .
$$

Doing so and proceeding as in the proof of Theorem $4.1^{*}$, we get the result.

Lemma 6.1. Suppose that $\mathcal{H}=\left\{a_{1} n+b_{1}, \ldots a_{k} n+b_{k}\right\}$ is an admissible $k$-tuple of linear forms and $q, a$ are two integers such that $\left(q, a_{i} a+b_{i}\right)=1$ for $1 \leq i \leq k$. Then the modified $k$-tuple $\tilde{\mathcal{H}}:=\left\{a_{1}(q m+a)+b_{1}, \ldots a_{k}(q m+a)+b_{k}\right\}$ is also admissible. Moreover,

$$
\mathfrak{S}(\tilde{\mathcal{H}})=\prod_{p \mid q}\left(1-\frac{\nu_{\mathcal{H}}(p)}{p}\right)^{-1} \mathfrak{S}(\mathcal{H}) \text {. }
$$

Proof. First, since $\mathcal{H}$ is admissible, we have $\left(a_{i}, b_{i}\right)=1$ for $1 \leq i \leq k$. Fix a prime $p$. We need to show that $\nu_{\tilde{\mathcal{H}}}(p)<p$. For a fixed $i$ we have either $p \mid a_{i}$, in which case $p \nmid b_{i}$ so $a_{i} n+b_{i} \not \equiv 0 \bmod p$, or $p \nmid a_{i}$, in which case the only solution to $a_{i} n+b_{i} \equiv 0 \bmod p$ is $n \equiv-a_{i}^{-1} b_{i}$. Hence, if $p \nmid a_{i}$, then there are only $\nu_{\mathcal{H}}(p)<p$ distinct possible values for $-a_{i}^{-1} b_{i} \bmod p$, thus regrouping these we can write

$$
\prod_{i=1}^{k}\left(a_{i} n+b_{i}\right) \equiv C \prod_{i: p \mid a_{i}} b_{i} \prod_{j=1}^{\nu_{\mathcal{H}}(p)}\left(n+k_{j}\right)^{e_{j}} \bmod p
$$

where the $k_{j}$ are distinct integers, $e_{j} \geq 1$ and $p \nmid C$. Using this and the fact that $\left(a_{i}, b_{i}\right)=1$, we get

$$
\prod_{i=1}^{k}\left(a_{i}(q m+a)+b_{i}\right) \equiv D \prod_{j=1}^{\nu_{\mathcal{H}}(p)}\left(q m+a+k_{j}\right)^{e_{j}} \bmod p
$$


with $p \nmid D$. If $p \nmid q$, then this has exactly $\nu_{\mathcal{H}}(p)<p$ solutions, therefore $\nu_{\tilde{\mathcal{H}}}(p)<p$. Otherwise, this becomes

$$
\prod_{i=1}^{k}\left(a_{i}(q m+a)+b_{i}\right) \equiv \prod_{i=1}^{k}\left(a_{i} a+b_{i}\right) \not \equiv 0 \bmod p
$$

since $\left(q, a_{i} a+b_{i}\right)=1$ for $1 \leq i \leq k$. We conclude that $\tilde{\mathcal{H}}$ is admissible. The calculation of $\mathfrak{S}(\tilde{\mathcal{H}})$ follows easily.

Proof of Theorem 2.5. Define $\mathcal{S}:=\emptyset$ and

$$
\mathbf{a}(n):=\prod_{\mathcal{L} \in \mathcal{H}} \Lambda(\mathcal{L}(n))=\Lambda\left(a_{1} n+b_{1}\right) \Lambda\left(a_{2} n+b_{2}\right) \cdots \Lambda\left(a_{k} n+b_{k}\right)
$$

In our context, some assumptions of Section 3.1 do not hold. The reason is that the asymptotic for $\mathcal{A}(x ; q, a)$ depends on $(q, \mathcal{P}(a ; \mathcal{H}))$ rather than depending only on $(q, a)$. The correct conjecture in this case is that for integers $a$ and $q$ such that $(q, \mathcal{P}(a ; \mathcal{H}))=1$, (see [13] $\left.]^{2}\right)$

$$
\mathcal{A}(x ; q, a) \sim \frac{\mathcal{A}(x)}{q \gamma(q)},
$$

with

$$
\gamma(q):=\prod_{p \mid q}\left(1-\frac{\nu_{\mathcal{H}}(p)}{p}\right) .
$$

This actually follows from the Hardy-Littlewood conjecture, by taking the modified $k$-tuple of linear forms $\tilde{\mathcal{L}}_{i}(m):=a_{i}(q m+a)+b_{i}=q a_{i} m+a a_{i}+b_{i}$, which is admissible if $\mathcal{H}$ is and $(q, \mathcal{P}(a ; \mathcal{H}))=1$ (see Lemma 6.1). Using this idea, we get that the assumption of (4) holding uniformly for $\left|a_{i}\right| \leq \mathbf{L}(x)^{1+\delta}$ implies Hypothesis $\left[3.1^{*}\right.$. We now prove an analogue of Proposition 5.1. Defining

$$
Z_{\mathcal{H}}(s):=\sum_{n} \frac{\mathbf{f}_{a}(n)}{n^{s+1} \gamma(n)}
$$

where

$$
\mathbf{f}_{a}(q):= \begin{cases}1 & \text { if }(\mathcal{P}(a ; \mathcal{H}), q)=1 \\ 0 & \text { otherwise }\end{cases}
$$

\footnotetext{
${ }^{2}$ Kawada imposes the additional condition that $R(\mathbf{b}):=\prod_{j=1}^{k}\left|a_{j}\right| \prod_{1 \leq i, j \leq k}\left|a_{i} b_{j}-a_{j} b_{i}\right|$ is non-zero. However, we assume that our linear forms are admissible, distinct and $a_{i} \geq 1$; one can show that this implies $R(\mathbf{b}) \neq 0$.
} 
one can compute that

$$
Z_{\mathcal{H}}(s)=\mathfrak{S}_{2}(s) \zeta(s+1) \zeta(s+2)^{k} Z_{0}(s)
$$

with

$$
\mathfrak{S}_{2}(s):=\prod_{p \mid \mathcal{P}(a ; \mathcal{H})}\left(1-\frac{1}{p^{s+1}}\right)\left(1+\frac{\nu_{\mathcal{H}}(p)}{p-\nu_{\mathcal{H}}(p)} \frac{1}{p^{s+1}}\right)^{-1}
$$

and

$$
Z_{0}(s):=\prod_{p}\left(1+\frac{\nu_{\mathcal{H}}(p)}{p-\nu_{\mathcal{H}}(p)} \frac{1}{p^{s+1}}\right)\left(1-\frac{1}{p^{s+2}}\right)^{k}
$$

which converges for $\Re s>-3 / 2$. Note that $Z_{\mathcal{H}}(s)$ has a simple pole at $s=0$. Also, $\mathfrak{S}_{2}(s)$ has a zero of order $\omega(\mathcal{P}(a, \mathcal{H}))$ at the point $s=-1$, and $Z_{0}(-1)=\mathfrak{S}(\mathcal{H})^{-1}$, so $Z_{\mathcal{H}}(s)$ is of $\operatorname{order} \omega(\mathcal{P}(a, \mathcal{H}))-k$ at this point. The function

$$
\psi(s):=\frac{(2 M)^{s}-M^{s}}{s+1}+\left(\frac{x}{2 M}\right)^{s}-\left(\frac{x}{M}\right)^{s}
$$

vanishes to the second order at $s=0$. Combining all this information, we obtain by shifting the contour of integration to the left that

$$
\frac{1}{2 \pi i} \int_{(1)} Z_{\mathcal{H}}(s) \psi(s) \frac{d s}{s}=\frac{1}{2 M}\left(\mu_{1-k}(a, M)(1+o(1))+O\left(\frac{1}{M^{\delta_{k}}}\right)\right)
$$

where

$$
\mu_{1-k}(a, M):= \begin{cases}-\frac{1}{2 \mathfrak{S}(\mathcal{H})} \frac{(\log M)^{k-\omega(\mathcal{P}(a ; \mathcal{H}))}}{(k-\omega(\mathcal{P}(a ; \mathcal{H})) !} \prod_{p \mid \mathcal{P}(a ; \mathcal{H})} \frac{p-\nu_{\mathcal{H}}(p)}{p} \log p & \text { if } \omega(\mathcal{P}(a ; \mathcal{H})) \leq k \\ 0 & \text { otherwise }\end{cases}
$$

and $\delta_{k}>0$ is a small real number (one can take $\delta_{k}=\frac{1}{2+k}$ ). We conclude by proceeding as in the proof of Theorem $4.1^{*}$.

In the case of twin primes (that is $\mathbf{a}(n):=\Lambda(n) \Lambda(n+2)$ ), we give an explicit description of all integers $a \geq-1$ (without loss of generality since $-a(-a+2)=a(a-2)$ ) for which $\mu_{-1}(a, M) \neq 0$ (note the occurrence of Mersenne and Fermat primes): 


\begin{tabular}{|c|c|c|}
\hline$a$ & $a(a+2)$ & $\omega(a(a+2))$ \\
\hline-1 & -1 & 0 \\
1 & 3 & 1 \\
2 & 8 & 1 \\
$p^{e}, p \neq 2: p^{e}+2=q^{f}$ & $p^{e} q^{f}$ & 2 \\
$2^{e}: 2^{e-1}+1=q^{f}$ & $2^{e+1}\left(2^{e-1}+1\right)$ & 2 \\
$2^{e}-2: 2^{e-1}-1=q^{f}$ & $2^{e+1}\left(2^{e-1}-1\right)$ & 2 \\
\hline
\end{tabular}

Proof of Theorem 2.6. Define $\mathcal{S}:=\emptyset$ and $\mathbf{L}(x):=(\log x)^{1-\delta}$. We split the proof in two cases, depending on the size of $y$.

Case 1: $\log y \leq(\log M)^{\frac{1}{2}-\delta}$. The fundamental lemma of combinatorial sieve (see [5]) gives the following estimate, in the range $2 \leq y \leq x^{o(1)}$ :

$$
\mathcal{A}(x, y)=x \prod_{p \leq y}\left(1-\frac{1}{p}\right)(1+E(x, y)),
$$

where $E(x, y) \ll x^{-\frac{1}{3}}$ for $2 \leq y<\frac{(\log x)^{2}}{16}$, and $E(x, y) \ll u^{-u}(\log y)^{3}$ for $\frac{(\log x)^{2}}{16} \leq y \leq x$, with the usual notation $u:=\frac{\log x}{\log y}\left(\right.$ so $y^{u}=x$ ). This shows that Hypothesis 3.2 holds. One shows that

$$
\mathcal{A}_{d}(x, y):=\sum_{\substack{n \leq x \\ d \mid n}} \mathbf{a}_{y}(n)= \begin{cases}\mathcal{A}\left(\frac{x}{d}, y\right) & \text { if } p \mid d \Rightarrow p \geq y \\ 0 & \text { else }\end{cases}
$$

so we have $\mathcal{A}_{d}(x, y)=\mathcal{A}\left(\frac{\mathbf{h}_{y}(d)}{d} x, y\right)$, where

$$
\mathbf{h}_{y}(d):= \begin{cases}1 & \text { if } p \mid d \Rightarrow p \geq y \\ 0 & \text { else. }\end{cases}
$$

Wolke [18] as shown a Bombieri-Vinogradov theorem for this sequence, which states that for any $A>0$, there exists $B=B(A)$ such that for any $Q \leq x^{\frac{1}{2}} / \log ^{B} x$, we have, uniformly in the range $y \leq \sqrt{x}$,

$$
\sum_{q \leq Q} \max _{(a, q)=1} \max _{z \leq x}\left|\sum_{\substack{n \leq z \\ n \equiv a \bmod q}} \mathbf{a}_{y}(n)-\frac{1}{\phi(q)} \sum_{\substack{n \leq z \\(n, q)=1}} \mathbf{a}_{y}(n)\right| \ll \frac{x}{\log ^{A} x} .
$$


(Notice that if $(a, q)>1$, then $\mathcal{A}(x, y ; q, a)$ is bounded.) We will only use this for $Q=2 \mathbf{L}(x)$, so from now on we suppose that $q \leq 2(\log x)^{1-\delta}$. Arguing as in Section 3.1, we have for $\frac{x}{2 \mathbf{L}(x)} \leq z \leq x$ that

$$
\begin{aligned}
\frac{1}{\phi(q)} \sum_{\substack{n \leq z \\
(n, q)=1}} \mathbf{a}_{y}(n) & =\frac{1}{\phi(q)} \sum_{d \mid q} \mu(d) \mathcal{A}_{d}(z, y)=\frac{1}{\phi(q)} \sum_{d \mid q} \mu(d) \mathcal{A}\left(\frac{\mathbf{h}_{y}(d)}{d} z, y\right) \\
& =\frac{\mathcal{A}(z, y)}{\phi(q)} \sum_{d \mid q} \frac{\mathbf{h}_{y}(d) \mu(d)}{d}\left(1+E_{d ; q}(z, y)\right)=\frac{\mathcal{A}(z, y)}{q \gamma_{y}(q)}\left(1+O\left(x^{-\frac{1}{3}+o(1)}\right)\right)
\end{aligned}
$$

since by (36), in the range $d \leq q \leq(\log x)^{1-\delta}$ we have

$$
E_{d ; q}(z, y) \ll\left(\frac{d}{z}\right)^{\frac{1}{3}} \ll x^{-\frac{1}{3}+o(1)} .
$$

Summing this over $q \leq 2 \mathbf{L}(x)$ and using (37), we get that

$$
\sum_{q \leq 2 \mathbf{L}(x)} \max _{(a, q)=1} \max _{\frac{x}{2 \mathbf{L}(x)} \leq z \leq x}\left|\mathcal{A}(z, y ; q, a)-\frac{\mathcal{A}(z, y)}{q \gamma_{y}(q)}\right| \ll \frac{\mathcal{A}(x, y)}{\mathbf{L}(x)^{1+\delta}} .
$$

Having a Bombieri-Vinogradov theorem in hand, we now prove an analogue of Proposition 5.1. A straightforward computation shows that

$$
\begin{aligned}
Z_{\mathcal{A}}(s):=\sum_{\substack{n \geq 1 \\
(n, a)=1}} \frac{1}{n^{s+1} \gamma_{y}(n)} & =\zeta(s+1) \prod_{p \mid a}\left(1-\frac{1}{p^{s+1}}\right) \prod_{\substack{p \nmid a \\
p<y}}\left(1+\frac{1}{(p-1) p^{s+1}}\right) \\
& =\mathfrak{S}_{a}(s) \zeta(s+1) \zeta(s+2) Z_{11}(s) \prod_{p \geq y}\left(1+\frac{1}{(p-1) p^{s+1}}\right)^{-1},
\end{aligned}
$$

where

$$
\begin{aligned}
& \mathfrak{S}_{a}(s):=\prod_{p \mid a}\left(1-\frac{1}{p^{s+1}}\right)\left(1+\frac{1}{(p-1) p^{s+1}}\right)^{-1}, \\
& Z_{11}(s):=\prod_{p}\left(1+\frac{1}{(p-1) p^{s+2}}-\frac{1}{(p-1) p^{2 s+3}}\right) .
\end{aligned}
$$

We will now use representation (39). Representation (40) will be useful for larger values of $y$, since then $\prod_{p<y}\left(1-\frac{1}{p^{s+2}}\right)^{-1}$ behaves like $\zeta(s+2)$ on the line $\Re(s)=-1+\frac{1}{\log M}$. Note that by (39), $Z_{\mathcal{A}}(s)$ is defined on the whole complex plane, except at $s=0$. As before, we need to compute the integral

$$
I:=\frac{1}{2 \pi i} \int_{(2)} Z_{\mathcal{A}}(s) \psi(s) \frac{d s}{s},
$$


where

$$
\psi(s):=\frac{(2 M)^{s}-M^{s}}{s+1}+\left(\frac{x}{2 M}\right)^{s}-\left(\frac{x}{M}\right)^{s},
$$

which has a double zero at $s=0$, so

$$
\begin{aligned}
I & =\frac{1}{2 \pi i} \int_{(-1 / 2)} Z_{\mathcal{A}}(s) \psi(s) \frac{d s}{s} \\
& =\frac{1}{2 \pi i} \int_{(-1 / 2)} Z_{\mathcal{A}}(s)\left((2 M)^{s}-M^{s}\right) \frac{d s}{s(s+1)}+O_{a, \epsilon}\left(\left(\frac{M}{x}\right)^{\frac{1}{2}-\epsilon} \log y\right),
\end{aligned}
$$

by the same arguments as in Lemma 5.12 (and Merten's theorem). We now proceed as in the proof of Proposition 5.9. Moving the contour of integration to $\Re(s)=\sigma=-1+\frac{1}{\log M}$ and using the bounds $Z_{\mathcal{A}}(\sigma+i t) \ll_{\epsilon}(|t|+1)^{\frac{1}{2}+\epsilon} \log y$ and $Z_{\mathcal{A}}^{\prime}(\sigma+i t) \ll_{\epsilon}(|t|+1)^{\frac{1}{2}+\epsilon}(\log y)^{2}$ for $|t| \geq 2$ (by Cauchy's theorem for the derivatives), we can deduce that

$$
I=\frac{2 \mu_{y}(a, M)-\mu_{y}(a, 2 M)}{2 M}\left(1+O_{a}\left(\frac{(\log y)^{2} \log \log M}{\log M}\right)\right)+o(1),
$$

where

$$
\mu_{y}(a, M):= \begin{cases}-\frac{1}{2} \prod_{p<y}\left(1-\frac{1}{p}\right)^{-1} & \text { if } a= \pm 1 \\ 0 & \text { else. }\end{cases}
$$

We conclude the proof in the same lines as that of Theorem $4.11^{*}$. Case 2: $\mathbf{L}^{(1+\delta) \log \log \mathbf{L}} \leq$ $y \leq \sqrt{x}$. Note that it is sufficient to consider this range, since $\mathbf{L}^{(1+\delta) \log \log \mathbf{L}}<(\log x)^{\log \log \log x}$. We have

$$
\mathcal{A}(x, y)=\frac{x \omega(u)}{\log y}\left(1+O\left(\frac{1}{\log y}\right)\right),
$$

where $u:=\frac{\log x}{\log y}$ and $\omega(u)$ is Buchstab's function (see Théorème III.6.4 of [17]). Therefore, we can use the properties of $\omega(u)$ to show that in the range $\frac{1}{\mathbf{L}(x)} \leq z \leq 1+\delta$,

$$
\frac{\mathcal{A}(z x, y)}{A(x, y)}=z \frac{\omega\left(u-O\left(\frac{\log \mathbf{L}}{\log y}\right)\right)}{\omega(u)}\left(1+O\left(\frac{1}{\log y}\right)\right)=z\left(1+O\left(\frac{\log \mathbf{L}}{\log y}\right)\right),
$$

hence Hypothesis 3.2 holds. Now, since $q \leq 2 \mathbf{L}(x)<y$, we have the equality

$$
\frac{1}{\phi(q)} \sum_{\substack{n \leq x \\(n, q)=1}} \mathbf{a}_{y}(n)=\frac{1}{\phi(q)} \sum_{n \leq x} \mathbf{a}_{y}(n)=\frac{\mathcal{A}(x, y)}{q \gamma_{y}(q)}
$$


thus using (37) we conclude that Hypothesis $3.1^{*}$ holds. We now turn to an analogue of Proposition 5.1, which we prove using (40). We need an estimate for

$$
\begin{aligned}
I & =\frac{1}{2 \pi i} \int_{(-1 / 2)} Z_{\mathcal{A}}(s) \psi(s) \frac{d s}{s} \\
& =\frac{1}{2 \pi i} \int_{(-1 / 2)} Z_{\mathcal{A}}(s)\left((2 M)^{s}-M^{s}\right) \frac{d s}{s(s+1)}+O_{a, \epsilon}\left(\left(\frac{M}{x}\right)^{\frac{1}{2}-\epsilon}\right),
\end{aligned}
$$

since on the line $\sigma=-1+\frac{1}{\log M}$, we have the bound

$$
\prod_{p \geq y}\left(1+\frac{1}{(p-1) p^{s+1}}\right)^{-1} \ll \prod_{p \geq \mathbf{L}^{(1+\delta) \log \log \mathbf{L}}}\left(1+\frac{C_{1}}{p(\log p)^{1+\delta}}\right) \ll 1
$$

and similarly for the derivative of this product. We now study the function $Z(s):=\frac{Z_{\mathcal{A}}(s)}{s(s+1)}$. Using the bounds we just proved, we get that for $s=-1+\frac{1}{\log M}+i t$ with $|t| \geq 2$,

$$
|Z(s)|,\left|Z^{\prime}(s)\right| \ll_{\epsilon}(|t|+1)^{-\frac{3}{2}+\epsilon}
$$

If $\omega(a) \geq 2$, then $Z(s)$ and $Z^{\prime}(s)$ are bounded near $s=-1$ and we conclude that $I=o(1)$. If $\omega(a)=1$, then we define

$$
Z_{12}(s):=Z(s)-\frac{c(M, y)}{s+1}
$$

where

$$
c(M, y):=-\frac{1}{2} \frac{\phi(a)}{a} \prod_{p \mid a} \log p \prod_{p \geq y}\left(1+\frac{1}{(p-1) p^{\frac{1}{\log M}}}\right)^{-1} .
$$

One sees that for $s$ close to -1 with $\Re(s)=\frac{1}{\log M}$,

$$
\prod_{p \geq y}\left(1+\frac{1}{(p-1) p^{s+1}}\right)^{-1}=(1+O(|s+1|)) \prod_{p \geq y}\left(1+\frac{1}{(p-1) p^{\frac{1}{\log M}}}\right)^{-1}
$$

hence $\left|Z_{12}^{\prime}(s)\right| \ll \frac{1}{|s+1|}$, and thus

$$
I=-\frac{1}{2} \frac{\phi(a)}{a} \prod_{p \mid a} \log p \prod_{p \geq y}\left(1+\frac{1}{(p-1) p^{\frac{1}{\log M}}}\right)^{-1}(1+o(1)) .
$$

If $a= \pm 1$, then we take $Z_{13}(s):=Z(s)-\frac{c(M, y)}{(s+1)^{2}}$, and since $Z_{13}(s) \ll \frac{1}{|s+1|}$, we get that (41) holds. Finally, in our range of $y$,

$$
\prod_{p \geq y}\left(1+\frac{1}{(p-1) p^{\frac{1}{\log M}}}\right)^{-1}=1+O\left(\frac{1}{\log y}\right) .
$$




\section{Appendix A. Generalities on Binary quAdratic Forms}

In this section we review several classical facts about the distribution of positive definite binary quadratic forms $Q(x, y)=\alpha x^{2}+\beta x y+\gamma y^{2}$ in arithmetic progressions. We recall the notations $d=\beta^{2}-4 \alpha \gamma, \mathcal{S}=\{p \mid 2 d\}, \chi_{d}=\left(\frac{4 d}{.}\right)$ and

$$
R_{a}(q)=\#\{1 \leq x, y \leq q: Q(x, y) \equiv a \bmod q\}
$$

Lemma A.1. The function $R_{a}(q)$ is multiplicative as a function of $q$.

Proof. Define $S_{a}(q):=\left\{(x, y) \in(\mathbb{Z} \cap[1, q])^{2}: Q(x, y) \equiv a \bmod q\right\}$ and let $q_{1}, q_{2}$ be two coprime integers. The "reduction mapping"

$$
\begin{aligned}
S_{a}\left(q_{1} q_{2}\right) & \rightarrow S_{a}\left(q_{1}\right) \times S_{a}\left(q_{2}\right) \\
(x, y) \bmod q_{1} q_{2} & \mapsto\left((x, y) \bmod q_{1},(x, y) \bmod q_{2}\right)
\end{aligned}
$$

is a bijection by the Chinese remainder theorem.

Lemma A.2. Take $Q(x, y):=x^{2}-d y^{2}$ with $d \equiv-1 \bmod 4$, and let $a \neq 0$ be a fixed integer such that $(a, 2 d)=1$. We have that

$$
\frac{R_{a}(q)}{q^{2}}=\frac{\mathbf{f}_{a}(q)}{q \gamma(q)}
$$

where

$$
\gamma(q):=\prod_{p \mid q}\left(1-\frac{\chi_{d}(p)}{p}\right)^{-1}
$$

and $\mathbf{f}_{a}(q)$ is a multiplicative function defined on primes as follows.

For $p \nmid 2 a d, \mathbf{f}_{a}\left(p^{e}\right):=1$. For $p^{f} \|$ a with $f \geq 1$ (so $\left.p \nmid 2 d\right)$, 


$$
\mathbf{f}_{a}\left(p^{e}\right):= \begin{cases}e+1+\frac{1}{p-1} & \text { if } \chi_{d}(p)=1, e \leq f \\ f+1 & \text { if } \chi_{d}(p)=1, e>f \\ \frac{1}{p+1} & \text { if } \chi_{d}(p)=-1, e \leq f, 2 \nmid e \\ 1-\frac{1}{p+1} & \text { if } \chi_{d}(p)=-1, e \leq f, 2 \mid e \\ 0 & \text { if } \chi_{d}(p)=-1, e>f, 2 \nmid f \\ 1 & \text { if } \chi_{d}(p)=-1, e>f, 2 \mid f .\end{cases}
$$

For $p \mid 2 d($ so $p \nmid a)$,

$$
\mathbf{f}_{a}\left(p^{e}\right)= \begin{cases}1+\left(\frac{a}{p}\right) & \text { if } p \neq 2 \\ 1+\left(\frac{-4}{a}\right) & \text { if } p=2, e \geq 2 \\ 1 & \text { if } p=2, e=1 .\end{cases}
$$

Proof. By Lemma A.1, it is enough to show that for any prime $p$ and integer $e \geq 1$,

$$
\frac{R_{a}\left(p^{e}\right)}{p^{e}}=\frac{\mathbf{f}_{a}\left(p^{e}\right)}{\gamma(p)}
$$

First case: $p \nmid 2 d$.

We will proceed as in section 2.3 of [2], by using Gauss sums. Writing $e(n):=e^{2 \pi i n}$,

$$
\begin{aligned}
R_{a}\left(p^{e}\right) & =\frac{1}{p^{e}} \sum_{1 \leq m \leq p^{e}} e\left(-m \frac{a}{p^{e}}\right)\left(\sum_{1 \leq x \leq p^{e}} e\left(m \frac{x^{2}}{p^{e}}\right)\right)\left(\sum_{1 \leq y \leq p^{e}} e\left(-m d \frac{y^{2}}{p^{e}}\right)\right) \\
& =p^{e}+\frac{1}{p^{e}} \sum_{1 \leq m \leq p^{e}-1} e\left(-m \frac{a}{p^{e}}\right) g\left(m ; p^{e}\right) g\left(-m d ; p^{e}\right)
\end{aligned}
$$

where $g(m ; q):=\sum_{n=1}^{q} e\left(m n^{2} / q\right)$ is a Gauss sum. We have the following properties (see [1]):

$$
\begin{array}{ll}
\text { If } q \text { is odd, then } & g(1 ; q)^{2}=\left(\frac{-1}{q}\right) q . \\
\text { If }(q, m)=1, \text { then } & g(m ; q)=\left(\frac{m}{q}\right) g(1 ; q) .
\end{array}
$$


As for Ramanujan sums, (see for example (3.3) of [12])

$$
\sum_{\substack{m=1 \\(m, q)=1}}^{q} e(m a / q)=\phi(q) \frac{\mu(q /(q, a))}{\phi(q /(q, a))} .
$$

Using these properties, we compute

$$
\begin{aligned}
R_{a}\left(p^{e}\right) & =p^{e}+\frac{1}{p^{e}} \sum_{g=1}^{e} \sum_{\substack{1 \leq m \leq p^{e}-1 \\
p^{e-g} \| m}} e\left(-m \frac{a}{p^{e}}\right) g\left(m ; p^{e}\right) g\left(-m d ; p^{e}\right) \\
& =p^{e}+\frac{1}{p^{e}} \sum_{g=1}^{e} \sum_{\substack{1 \leq m^{\prime} \leq p^{g}-1 \\
p \nmid m^{\prime}}} e\left(-m^{\prime} \frac{a}{p^{g}}\right) p^{2 e-2 g} g\left(m^{\prime} ; p^{g}\right) g\left(-m^{\prime} d ; p^{g}\right) \\
& =p^{e}+p^{e} \sum_{g=1}^{e}\left(\frac{d}{p^{g}}\right) p^{-g} \sum_{\substack{1 \leq m^{\prime} \leq p^{g}-1 \\
p \nmid m^{\prime}}} e\left(-m^{\prime} \frac{a}{p^{g}}\right) \\
& =p^{e}+p^{e} \sum_{g=1}^{e}\left(\frac{d}{p}\right)^{g}\left(1-\frac{1}{p}\right) \frac{\mu\left(p^{g} /\left(p^{g}, a\right)\right)}{\phi\left(p^{g} /\left(p^{g}, a\right)\right)}
\end{aligned}
$$

which shows (after a straightforward computation) that (44) holds for $p \nmid 2 d$.

Second case: $p \mid 2 d, p \neq 2$.

In this case we have that $p \nmid a$, since $(a, \mathcal{S})=1$. The number of solutions of $x^{2}-d y^{2} \equiv$ $a \bmod p$ is exactly $p\left(1+\left(\frac{a}{p}\right)\right)$. Moreover, such a solution must satisfy $x \not \equiv 0 \bmod p$, thus by Hensel's lemma we obtain that

$$
\frac{R_{a}\left(p^{e}\right)}{p^{e}}=1+\left(\frac{a}{p}\right)
$$

Third case: $p=2$.

In this case, $2 \nmid a$. We have that $R_{a}(2)=2$. Reducing the equation $x^{2}-d y^{2} \equiv a \bmod 2^{e}$ (using that $d \equiv-1 \bmod 4$ ), we get

$$
x \not \equiv y \bmod 2, \quad x^{2}+y^{2} \equiv a \bmod 4,
$$

which shows that there are no solutions if $a \equiv 3 \bmod 4$. Suppose now that $a \equiv 1 \bmod 4$. For $e \geq 3$, an odd integer is a square $\bmod 2^{e}$ if and only if it is congruent to $1 \bmod 8$; in fact we have the following isomorphism:

$$
\left(\mathbb{Z} / 2^{e} \mathbb{Z}\right)^{\times} \simeq \mathbb{Z} / 2 \mathbb{Z} \times \mathbb{Z} / 2^{e-2} \mathbb{Z}
$$


Using these well-known facts, we find the number of solutions to $x^{2}-d y^{2} \equiv a$ mod $2^{e}$ such that $x$ is odd is

$$
\begin{aligned}
& =4 \#\left\{y \bmod 2^{e}: d y^{2}+a \equiv 1 \bmod 8\right\} \\
& =2^{e-1} \#\left\{y \bmod 8: y^{2} \equiv d^{-1}(1-a) \bmod 8\right\}=2^{e}
\end{aligned}
$$

since $d^{-1}(1-a) \equiv 0,4 \bmod 8$. Now the number of solutions of $x^{2}-d y^{2} \equiv a \bmod 2^{e}$ such that $x$ is even is just the number of solutions of $y^{2}-d^{-1} x^{2} \equiv-d^{-1} a \bmod 2^{e}$ such that $y$ is odd, which as we have shown (and using that $-d^{-1} \equiv 1 \bmod 4$ ) is equal to $2^{e}$. We conclude that

$$
\frac{R_{a}\left(2^{e}\right)}{2^{e}}= \begin{cases}2 & \text { if } a \equiv 1 \bmod 4 \\ 0 & \text { if } a \equiv 3 \bmod 4\end{cases}
$$

Lemma A.3. Take $Q(x, y):=\alpha x^{2}+\beta x y+\gamma y^{2}$ with $(\alpha, \beta, \gamma)=1$ and $d=\beta^{2}-4 \alpha \gamma \equiv$ $1,5,9,12,13 \bmod 16$. Let $a \neq 0$ be a fixed integer with $(a, 2 d)=1$. We have for $(q, 2 d)=1$ that

$$
\frac{R_{a}(q)}{q^{2}}=\frac{\mathbf{f}_{a}(q)}{q \gamma(q)}
$$

where

$$
\gamma(q):=\prod_{p \mid q}\left(1-\frac{\chi_{d}(p)}{p}\right)^{-1}
$$

and $\mathbf{f}_{a}(q)$ is defined as in Lemma A.2. Moreover, for $p \mid 2 d, p \neq 2$ (so $\left.p \nmid a\right)$,

$$
\frac{R_{a}\left(p^{e}\right)}{p^{e}}= \begin{cases}1+\left(\frac{\alpha a}{p}\right) & \text { if } p \neq 2, p \nmid \alpha \\ 1+\left(\frac{\gamma a}{p}\right) & \text { if } p \neq 2, p \nmid \gamma\end{cases}
$$

and

$$
\frac{R_{a}\left(2^{e}\right)}{2^{e}}= \begin{cases}1 & \text { if } 2 \mid \beta, e=1 \\ 1+\left(\frac{-4}{\alpha a}\right) & \text { if } 2 \mid \beta, 2 \nmid \alpha, e \geq 2 \\ 1+\left(\frac{-4}{\gamma a}\right) & \text { if } 2 \mid \beta, 2 \nmid \gamma, e \geq 2 \\ \frac{1}{2} & \text { if } 2 \nmid \beta, 2 \mid \alpha \gamma \\ \frac{3}{2} & \text { if } 2 \nmid \alpha \beta \gamma .\end{cases}
$$


Proof. First write $Q(x, y)$ in four different ways:

$$
\begin{aligned}
Q(x, y) & =\frac{1}{4 \alpha}\left((2 \alpha x+\beta y)^{2}-d y^{2}\right) \\
& =\frac{1}{\alpha}\left(\left(\alpha x+\frac{\beta}{2} y\right)^{2}-\frac{d}{4} y^{2}\right) \\
& =\frac{1}{4 \gamma}\left((\beta x+2 \gamma y)^{2}-d x^{2}\right) \\
& =\frac{1}{\gamma}\left(\left(\gamma y+\frac{\beta}{2} x\right)^{2}-\frac{d}{4} x^{2}\right) .
\end{aligned}
$$

We will split in five distinct cases.

Case 1: $p \nmid 2 \alpha$. In this case, we use the representation (50). Note that the mapping $\phi_{y}: x \mapsto 2 \alpha x+\beta y$ is an automorphism of $\mathbb{Z} / p^{e} \mathbb{Z}$, so

$$
R_{a}\left(p^{e}\right)=\#\left\{1 \leq x, y \leq p^{e}: x^{2}-d y^{2} \equiv 4 \alpha a \bmod p^{e}\right\}
$$

Going through the proof of Lemma A.2, we see that

$$
\frac{R_{a}\left(p^{e}\right)}{p^{e}}=\frac{\mathbf{f}_{4 \alpha a}\left(p^{e}\right)}{\gamma(p)}=\frac{\mathbf{f}_{\alpha a}\left(p^{e}\right)}{\gamma(p)} \quad\left(=\frac{\mathbf{f}_{a}\left(p^{e}\right)}{\gamma(p)} \text { if } p \nmid d\right) .
$$

Case 2: $p \nmid 2 \gamma$. In this case, we proceed in an analogous way to the first case, using the representation (52) to get that

$$
\frac{R_{a}\left(p^{e}\right)}{p^{e}}=\frac{\mathbf{f}_{4 \gamma a}\left(p^{e}\right)}{\gamma(p)}=\frac{\mathbf{f}_{\gamma a}\left(p^{e}\right)}{\gamma(p)} \quad\left(=\frac{\mathbf{f}_{a}\left(p^{e}\right)}{\gamma(p)} \text { if } p \nmid d\right) .
$$

Case 3: $p|\alpha, p| \gamma, p \neq 2$. In this case $p \nmid \beta$, so $p \nmid d$. Writing $X:=x+y$ and $Y:=y$, we compute that

$$
\alpha X^{2}+\beta X Y+\gamma Y^{2}=\alpha x^{2}+(2 \alpha+\beta) x y+(\alpha+\beta+\gamma) y^{2}=: \alpha^{\prime} x^{2}+\beta^{\prime} x y+\gamma^{\prime} y^{2} .
$$

We have $p \mid \alpha^{\prime}, p \nmid \beta^{\prime}$ and $p \nmid \gamma^{\prime}$, which reduces the problem to Case 2 , and so

$$
\frac{R_{a}\left(p^{e}\right)}{p^{e}}=\frac{\mathbf{f}_{(\alpha+\beta+\gamma) a}\left(p^{e}\right)}{\gamma(p)}=\frac{\mathbf{f}_{a}\left(p^{e}\right)}{\gamma(p)}
$$

Case 4.1: $p=2,2 \mid \beta$. In this case, $d \equiv 0 \bmod 4$. We have that either $2 \nmid \alpha$, or $2 \nmid \gamma$. In the first event we use representation (151), which gives

$$
R_{a}\left(2^{e}\right)=\#\left\{1 \leq x, y \leq 2^{e}: x^{2}-d^{\prime} y^{2} \equiv \alpha a \bmod 2^{e}\right\}
$$


with $d^{\prime}:=\frac{d}{4} \equiv-1 \bmod 4$. Going back to the proof of Lemma A.2, we get that

$$
\frac{R_{a}\left(2^{e}\right)}{2^{e}}=\mathbf{f}_{\alpha a}\left(2^{e}\right)
$$

In the event that $2 \nmid \gamma$, the result is

$$
\frac{R_{a}\left(2^{e}\right)}{2^{e}}=\mathbf{f}_{\gamma a}\left(2^{e}\right)
$$

Note that if $2 \nmid \alpha \gamma$, then since $\frac{d}{4} \equiv-1 \bmod 4$, we have $\alpha \equiv \gamma \bmod 4$, so

$$
\mathbf{f}_{\alpha a}\left(2^{e}\right)=\mathbf{f}_{\gamma a}\left(2^{e}\right)
$$

Case 4.2: $p=2,2 \nmid \beta$. In this case, $2 \nmid d$ and $2 \nmid a$. An easy application of Hensel's lemma in either of the variables $x$ or $y$ (since one of them has to be odd) yields

$$
\frac{R_{a}\left(2^{e}\right)}{2^{e}}=\frac{R_{a}(2)}{2}
$$

and all the possibilities are contained in the following table.

\begin{tabular}{|c|c|c|c|}
\hline$\alpha \bmod 2$ & $\beta \bmod 2$ & $\gamma \bmod 2$ & $R_{a}(2)$ \\
\hline 0 & 1 & 0 & 1 \\
0 & 1 & 1 & 1 \\
1 & 1 & 0 & 1 \\
1 & 1 & 1 & 3 \\
\hline
\end{tabular}

\section{REFERENCES}

[1] Bruce C. Berndt, Ronald J. Evans, Kenneth S. Williams, Gauss and Jacobi sums. Canadian Mathematical Society Series of Monographs and Advanced Texts. A Wiley-Interscience Publication. John Wiley \& Sons, Inc., New York, 1998. xii+583 pp. ISBN: 0-471-12807-4

[2] Valentin Blomer, Jörg Brüdern and Rainer Dietmann, Sums of smooth squares. Compos. Math. 145 (2009), no. 6, 1401-1441.

[3] Enrico Bombieri, John B. Friedlander and Henryk Iwaniec, Primes in arithmetic progressions to large moduli. Acta Math. 156 (1986), no. 3-4, 203-251.

[4] Harold Davenport, Multiplicative number theory. Third edition. Revised and with a preface by Hugh L. Montgomery. Graduate Texts in Mathematics, 74. Springer-Verlag, New York, 2000. xiv+177 pp. ISBN: 0-387-95097-4 
[5] Nicolaas Govert De Bruijn, On the number of uncancelled elements in the sieve of Eratosthenes. Nederl. Akad. Wetensch., Proc. 53, (1950) 803-812

[6] Harold M. Edwards, Riemann's zeta function. Pure and Applied Mathematics, Vol. 58. Academic Press, New York-London, 1974. xiii +315 pp.

[7] Daniel Fiorilli, Residue classes containing an unexpected number of primes. preprint: arXiv:1009.2699v1 [math.NT].

[8] Andrew Granville, Kannan Soundararajan, An uncertainty principle for arithmetic sequences. Ann. of Math. (2) 165 (2007), no. 2, 593-635.

[9] Christopher Hooley, On the Barban-Davenport-Halberstam theorem. I Collection of articles dedicated to Helmut Hasse on his seventy-fifth birthday, III. J. Reine Angew. Math. 274/275 (1975), 206-223

[10] Martin N. Huxley, Exponential sums and the Riemann zeta function. V. Proc. London Math. Soc. (3) 90 (2005), no. 1, 1-41.

[11] Henryk Iwaniec, The half dimensional sieve. Acta Arith. 29 (1976), no. 1, 69-95.

[12] Henryk Iwaniec, Emmanuel Kowalski, Analytic number theory. American Mathematical Society Colloquium Publications, 53. American Mathematical Society, Providence, RI, 2004. xii +615 pp.

[13] Koichi Kawada, The prime $k$-tuplets in arithmetic progressions. Tsukuba J. Math. 17 (1993), no. 1, 43-57.

[14] Yoichi Motohashi, An induction principle for the generalization of Bombieri's prime number theorem. Proc. Japan Acad. 52 (1976), no. 6, 273-275.

[15] Vladimir Aleksandrovich Plaksin, Asymptotic formula for the number of solutions of an equation with primes. Izv. Akad. Nauk SSSR Ser. Mat. 45 (1981), no. 2, 321-397

[16] Vladimir Aleksandrovich Plaksin, Asymptotic formula for the number of representations of a natural number by a pair of quadratic forms, the arguments of one of which are primes. Izv. Akad. Nauk SSSR Ser. Mat. 48 (1984), no. 6, 1245-1265.

[17] Gérald Tenenbaum, Introduction à la théorie analytique et probabiliste des nombres. Deuxième édition, Cours Spécialisés, 1. Société Mathématique de France, Paris, 1995. xv+457 pp.

[18] Dieter Wolke, Über die mittlere Verteilung der Werte zahlentheoretischer Funktionen auf Restklassen. II. (German) Math. Ann. 204 (1973), 145-153.

Département de mathématiques et de statistique, Université de Montréal, CP 6128, succ. Centre-ville, Montréal, QC, Canada H3C 3J7

E-mail address: fiorilli@dms.umontreal.ca 